\title{
Approximations of steady periodic water waves in flows with constant vorticity.
}

\author{
A. Constantin* \\ K. Kalimeris ${ }^{\dagger}$ \\ O. Scherzer $\ddagger$
}

March 18, 2022

\begin{abstract}
We provide high-order approximations to periodic travelling wave profiles and to the velocity field and the pressure beneath the waves, in flows with constant vorticity over a flat bed.
\end{abstract}

Keywords: Travelling water waves, vorticity, velocity field, pressure. AMS Subject Classifications (2010): 35Q31, 35Q35, 76D33.

\section{Introduction}

Winds in offshore storms transmit energy to the ocean surface, creating waves. Once the waves leave the storm area they become organised into two-dimensional regular wave trains, in the form of a regular profile that propagates practically unchanged in a fixed direction. These periodic travelling waves are called 'swell' in oceanography. Their shape is influenced by the underlying currents. The most significant currents on areas of the continental shelf and in many coastal inlets are the tidal currents [20. These alternating horizontal movements of water are created by the gravitational pull of the moon, and to a lesser degree, the sun, on the earth's surface, being associated with the rise and fall of the tide: the current associated with a rising water level, called the flood, is directed towards the shore, while the current associated with a receding water level, called the ebb, is directed back out to sea. Flows of constant vorticity with a flat free surface provide adequate descriptions of pure tidal currents, cf. the discussion in [6], positive constant vorticity $\gamma>0$ being appropriate for the modelling of the ebb current and negative constant vorticity $\gamma<0$ for the flood current. A spectacular example of wave-current interaction is the Columbia River entrance, made by appreciable tidal currents one of the most hazardous navigational regions in the world since wave heights can easily be doubled in just a few hours [20. At this location, tidal velocities in excess of $2 \mathrm{~m} / \mathrm{s}$ are encountered and in winter wave heights in excess of $6 \mathrm{~m}$, up to 14-15 m, are common, cf. [21]. Note that moderate tidal currents reach speeds of up to $0.7 \mathrm{~m} / \mathrm{s}$, cf. [36],

\footnotetext{
*Faculty of Mathematics, University of Vienna, Oskar-Morgenstern-Platz 1, 1090 Vienna, Austria

${ }^{\dagger}$ Radon Institute of Computational and Applied Mathematics, Altenberger Str. 69, 4040 Linz, Austria

${ }^{\ddagger}$ Computational Science Center, University of Vienna, Oskar-Morgenstern-Platz 1, 1090 Vienna, Austria and Radon Institute of Computational and Applied Mathematics, Altenberger Str. 69, 4040 Linz, Austria
} 
while tidal currents with speeds of $5.5 \mathrm{~m} / \mathrm{s}$ are encountered in between the Scottish mainland and the Orkney Islands, in the Pentland Firth — see the data provided in [18].

In this paper we provide accurate approximations for the interaction of waves with underlying currents of constant vorticity in the absence of flow-reversal; for theoretical studies and numerical simulations of wave-current interactions with flow-reversal we refer to [14, 15, 35] and [27, 33], respectively. Let us specify that in a flow where both waves and currents are present, given velocity measurements at one point, the 'current' is defined as the average velocity, and the periodic components that vary around this average are ascribed to the wave motion. Since irrotational flows do not present flow-reversals as the underlying current must be uniform, one of our main purposes will be to highlight the effects of vorticity on the surface wave profile. This problem is of great practical relevance since the detection of non-uniform underlying currents from the surface wave pattern is particularly important to anticipate and avoid hazardous conditions, where possible. In addition to the wave profile, we will also provide approximations for the velocity profile and the pressure throughout the fluid. Not only is there a strong interplay of these flow characteristics that is very useful in qualitative studies - see [5, 13, but in practice information on the state of the sea surface is often gathered from subsurface pressure and/or velocity measurements, cf. the discussions in [3, 4, 7, 24, 28, 34]. Let us point out the following counter-intuitive fact: periodic travelling waves that propagate at the surface of water with a flat bed in a flow of constant vorticity must be symmetric if no flow-reversal occurs and if the wave profile is monotone between successive crests and troughs, cf. [8, 9, 25]. This means that an underlying non-uniform current of constant vorticity does not break the symmetry of irrotational wave trains, so that the manifestation of vorticity on the surface wave pattern must take subtler forms.

The fact that even when both the wave motion and the underlying current of constant vorticity are known with accuracy, their interaction produces a significantly different effect from that obtained by simply adding the effect of the waves and the currents considered separately, cf. the discussions in [29, 30, 31], shows the importance of dealing simultaneously with these two flow components. Linear theory (see the discussion in [6]) provides the dispersion relation

$$
c=u_{0}-\frac{\gamma \tanh (k d)}{2 k} \pm \frac{1}{2 k} \sqrt{\gamma^{2} \tanh ^{2}(k d)+4 g k \tanh (k d)}
$$

for the wave speed $c$, expressed in terms of the surface current speed $u_{0}$ in a flow of constant vorticity $\gamma$ in water of mean depth $d$; here $k=\frac{2 \pi}{L}$ is the frequency of a wave with wavelength $L$. Note that (1.1) permits us to understand the effect of vorticity on the propagation speed of waves of small amplitude (that are realistically described by linear theory). For example, from the point of view of a fixed observer noticing right-propagating waves that interact with a current with vanishing surface speed (a setting corresponding to $c>0$ and $u_{0}=0$ ), from (1.1) we infer that the propagation speed is

$$
c(\gamma)=-\frac{\gamma \tanh (k d)}{2 k}+\frac{1}{2 k} \sqrt{\gamma^{2} \tanh ^{2}(k d)+4 g k \tanh (k d)},
$$

irrespective of the sign of $\gamma$. In this setting the underlying current beneath the flat free surface $y=0$ and above the bed $y=-d$ is given by $u(y)=\gamma y$, so that $\gamma>0$ corresponds 
to an adverse current since everywhere beneath the surface the current velocity opposes the direction of wave propagation, $\gamma<0$ corresponds to a favourable current (the current velocity points everywhere beneath the surface in the direction of wave propagation), while the irrotational case $\gamma=0$ is characterized by the absence of an underlying current. A simple analysis of 1.2 confirms that

$$
c(-|\gamma|)>c(0)>c(|\gamma|)
$$

for every $\gamma \neq 0$. This means that favourable currents enhance the wave speed, while adverse currents reduce it. In contrast to this analysis of the wave speed, the first-order approximation that is pursued within the framework of linear theory is not conclusive in regard to the effect of underlying currents on the wave profiles: at first order, periodic travelling waves in flows of constant vorticity are sinusoidal (see [6]). Consequently, nonlinear effects have to be accounted for and higher-order approximations are needed. We present in this paper an approach that provides the second and third order asymptotic expansion of waves of small amplitude, expressed in terms of a suitable amplitude parameter $b$. Following this procedure, we can readily obtain other characteristics of the water flow, such as the velocity field and the pressure beneath the wave. The knowledge of accurate approximations for these wave characteristics allows us to compare the results with those for irrotational flows. In the last part of the work we illustrate these flow characteristics for several different types of wave-current interactions. Laboratory experiments and numerical simulations for irrotational waves are discussed in [1, 2, 32, while this type of studies for wave-current interactions in flows of constant vorticity were pursued in [11, 16, 22, 23, 29, 30]. The results presented below permit a more detailed analysis and numerical simulation.

\section{Mathematical Formulation}

In this paper we discuss two dimensional, periodic travelling waves with constant vorticity. We assume that the water is incompressible and inviscid, over a flat bed and acted upon by gravity $g$. In what follows we make no shallowness or small amplitude approximation. In order to avoid extended revision we refer to the recent works [6, 11, 12] for the mathematical formulation of the problem. In this section we make only a brief review of the problem, in order to provide sufficient terminology for the fundamental Boundary Value Problem (BVP) (2.10).

We are studying two-dimensional waves travelling at constant speed $c$. This means that in a two-dimensional frame moving with the constant speed $c$, the flow pattern - and, in particular, the shape of the surface of the fluid - does not change over time.

These assumptions, see [11], allow the definitions of the free surface profile by

$$
S=\{(x, y):-\pi<x<\pi \text { and } y=\eta(x)\},
$$

and the flat bed by

$$
B=\{(x, y):-\pi<x<\pi \text { and } y=-d\},
$$

with $d>0$, for the normalized wavelength $2 \pi$. 
Without loss of generality, the assumption that the waves oscillate around the flat free surface $y=0$ is made, i.e.,

$$
\int_{-\pi}^{\pi} \eta(x) d x=0
$$

We denote the velocity field of the flow by $(u(x, y), v(x, y))$, with $(x, y) \in \mathcal{D}$, where

$$
\mathcal{D}=\{(x, y):-\pi<x<\pi \text { and }-d<y<\eta(x)\} .
$$

In this paper we follow the assumption made in [12, that there are no flow-reversals, which is formulated as the condition

$$
u<c \text { throughout the fluid. }
$$

We denote the pressure in the fluid by $P(x, y)$, with $(x, y) \in \mathcal{D}$. Neglecting the effects of surface tension - a hypothesis that is appropriate for waves of moderate and large amplitude (see the discussion in [6] ) - we impose that the water pressure is constant on $S, P(x, \eta(x))=$ $P_{a t m}$, where $P_{a t m}$ is the atmospheric pressure.

Let us recall the following definitions:

- The vorticity of the flow is defined by

$$
\gamma:=u_{y}-v_{x}
$$

which for the rest of the paper we will assume constant.

- The relative mass flux ${ }^{1}$ is defined by

$$
p_{0}:=\int_{-d}^{\eta(x)}(u(x, y)-c) d y<0,
$$

which in fact is independent of $x$, see [11. Moreover, (2.4) and (2.2) show that $p_{0}$ is negative. This relation shows that the amount of water passing any vertical line is constant throughout $\mathcal{D}$.

- The stream function $\psi(x, y)$ is defined as the unique solution of the differential equations

$$
\psi_{x}=-v, \quad \psi_{y}=u-c \text { in } \overline{\mathcal{D}},
$$

subject to

$$
\psi(x,-d)=-p_{0}
$$

- In [12] it was proven that the expression

$$
\frac{(u-c)^{2}+v^{2}}{2}+g y+P-\gamma \psi
$$

equals a constant $E$ throughout $\mathcal{D}$. The constant $Q=E-P_{\text {atm }}$ is called the hydraulic head.

\footnotetext{
${ }^{1}$ The terminology 'relative' is due to the fact that $(u-c)$ is the relative horizontal velocity of the flow, with reference to the moving frame at speed $c$.
} 
We also recall some basic terminology, as used in [12]:

- Firstly, the Dubreil-Jacotin transformation maps the unknown domain $\mathcal{D}$ to the rectangle

$$
R=\left\{(q, p):-\pi<q<\pi, p_{0}<p<0\right\},
$$

The independent variables as they appear in the Dubreil-Jacotin transformation [17]

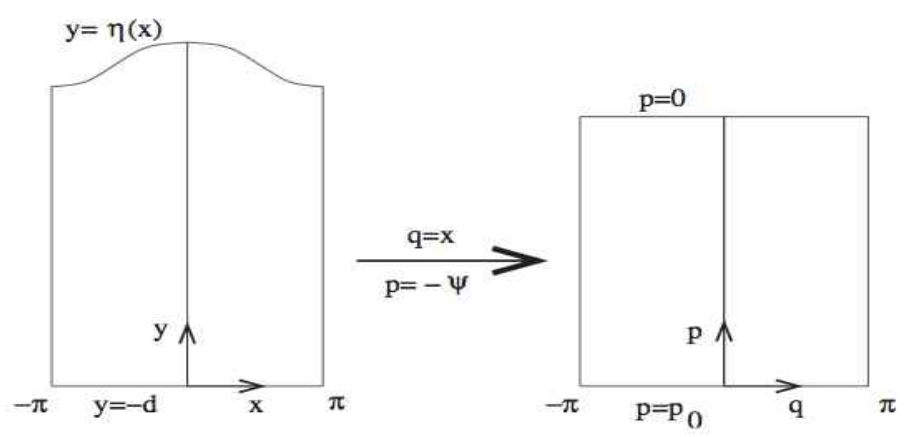

Figure 1: Dubreil-Jacotin transformation

are

$$
q=x, \quad p=-\psi .
$$

- Secondly, the height function of the wave above $B$ is defined as

$$
h(q, p)=y+d .
$$

This function satisfies the following nonlinear BVP on the rectangle $R$. We omit here the details of this construction, and refer to [12].

Definition 2.1. The constitutive equations for the height function $h(q, p)$, which is even and $2 \pi$-periodic in $q$, are the following:

$$
\left\{\begin{array}{l}
\mathcal{H}[h]:=\left(1+h_{q}^{2}\right) h_{p p}-2 h_{p} h_{q} h_{p q}+h_{p}^{2} h_{q q}-\gamma h_{p}^{3}=0 \quad \text { on } R, \\
\mathcal{B}_{0}[h]:=1+h_{q}^{2}(q, 0)+(2 g h-Q) h_{p}^{2}(q, 0)=0, \\
\mathcal{B}_{1}[h]:=h\left(q, p_{0}\right)=0
\end{array}\right.
$$

Moreover, the free boundary $S$ is given by the expression

$$
h(q, 0)=\eta(x)+d .
$$

In this equation $\gamma$ denotes the vorticity as defined in (2.3), which is assumed constant, being indicative of underlying currents. Vanishing vorticity is the property typical of uniform currents and a constant vorticity characterizes the linearly sheared tidal currents. We also recall that $g$ is the gravitational constant of acceleration, $Q$ is the hydraulic head and $p_{0}$ is the relative mass flux, introduced above. Moreover, we define the height of the wave as the maximal variation of the oscillations of the free surface, given by

$$
a:=\max _{q \in[-\pi, \pi]} h(q, 0)-\min _{q \in[-\pi, \pi]} h(q, 0)=h(0,0)-h(\pi, 0) .
$$


In fact, the BVP (2.10) is a reformulation of the Euler equations restricted to $\mathcal{D}$, under the assumptions made previously in this section.

In the following subsections we discuss some results from [12], and provide a new point of view, which allows a generalization of these results.

\subsection{Laminar solutions}

The laminar flows are readily obtained as the $q$-independent solutions of the boundary-value problem 2.10), given by the following formula

$$
H(p ; \lambda)=\frac{2\left(p-p_{0}\right)}{\sqrt{\lambda-2 \gamma p}+\sqrt{\lambda-2 \gamma p_{0}}}, \quad p_{0} \leq p \leq 0,
$$

provided that the parameter $\lambda>0$ satisfies the equation

$$
Q=\lambda+\frac{4 g\left|p_{0}\right|}{\sqrt{\lambda}+\sqrt{\lambda-2 \gamma p_{0}}}
$$

\subsection{Solutions of the linearised problem}

Apart from the laminar flows one can obtain the solution of the linearised problem. For this, the BVP 2.10 is linearised around the laminar flow $H(p ; \lambda)$. For the specific value $\lambda_{*}$ that satisfies the dispersion equation

$$
\frac{\lambda}{g-\gamma \sqrt{\lambda}}+\tanh \left(\frac{2 p_{0}}{\sqrt{\lambda}+\sqrt{\lambda-2 p_{0} \gamma}}\right)=0,
$$

an existence result for the solution of this BVP is presented in [12]. Moreover, the linearised solution is given by $\hat{h}(q, p ; b)=H\left(p ; \lambda_{*}\right)+b m(q, p)$, where

$$
m(q, p)=\frac{\sqrt{\lambda_{*}-2 p_{0} \gamma}}{\sqrt{\lambda_{*}-2 p \gamma}} \sinh \left(\frac{2\left(p-p_{0}\right)}{\sqrt{\lambda_{*}-2 \gamma p}+\sqrt{\lambda_{*}-2 \gamma p_{0}}}\right) \cos q .
$$

In our investigation we start from this result and we obtain an extension of it by making a two-fold interpretation.

Firstly, we interpret the function

$$
\hat{h}(q, p ; b)=H\left(p ; \lambda_{*}\right)+b m(q, p),
$$

as a perturbation of a laminar solution of the system (2.10), in the following sense: the system 2.10 is satisfied up to order $b^{2}$, i.e.,

$$
\mathcal{H}[\hat{h}](q, p)=\mathcal{O}\left(b^{2}\right), \mathcal{B}_{0}[\hat{h}](q)=\mathcal{O}\left(b^{2}\right) \text { and } \mathcal{B}_{1}[\hat{h}](q)=0
$$

and the height of the water wave is of order $b$, i.e,

$$
\hat{h}(0,0 ; b)-\hat{h}(\pi, 0 ; b)=b[m(0,0)-m(\pi, 0)]=\mathcal{O}(b) .
$$

Having in mind that the wave height vanishes for laminar flows, we can view the expression (2.16) as an approximation of small amplitude water waves.

Secondly, we can regard this expression as the first order asymptotic expansion of the exact solution to the BVP (2.10), which leads to the natural question of finding higher-order terms. This point of view is motivated by a bifurcation argument, provided in [12] and discussed below. 


\subsection{Bifurcation}

We define the curve that represents the laminar flows

$$
\mathcal{T}=\{(Q(\lambda), H(p ; \lambda)): \lambda>0\},
$$

with $Q$ and $H$ given by 2.13 ) and $(2.12$, respectively.

Restricting our attention to rotational flows with constant vorticity $\gamma$, in [12] it was proven that near this curve, as the parameter $\lambda$ varies, there are generally no genuine waves, except at critical values $\lambda=\lambda_{*}$ determined by the dispersion relation (2.14). Near this bifurcating laminar flow $H^{*}$, we have two solution curves: one laminar solution curve $\lambda \mapsto H(p ; \lambda)$, where $\lambda$ and $Q$ are related by (2.13), and one non-laminar solution curve $Q \mapsto h(q, p ; Q)$ such that $h_{q} \not \equiv 0$ unless $h=H^{*}$, see Figure 2,

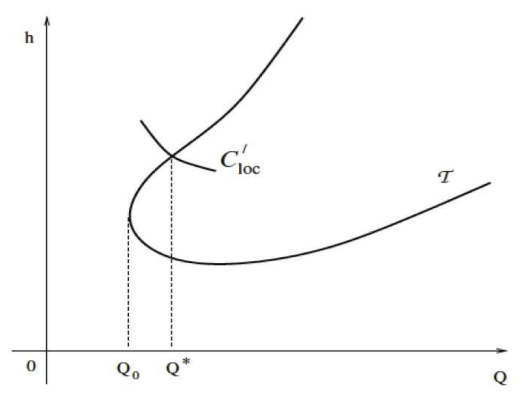

Figure 2: Bifurcation on the curve of laminar flows.

\subsection{Higher order approximation}

Using the above argument we observe that the variation of the parameter $Q$ (which is the hydraulic head of the flow) about the uniquely determined value $Q^{*}$ implies an approximation of non-laminar flows, in the following sense. Consider the approximation for the hydraulic head of the flow,

$$
Q \approx Q^{(m)}=Q(b)=Q^{*}+\sum_{n=1}^{m} Q_{n} b^{n},
$$

and the approximation for the height function $h(q, p ; Q)$,

$$
h(q, p ; Q) \approx h^{(m)}(q, p ; b)=\sum_{n=0}^{m} h_{n}\left(q, p ; Q^{*}\right) b^{n},
$$

with $h_{0}\left(q, p ; Q^{*}\right) \equiv H\left(p, \lambda_{*}\right)$.

Our goal is to determine $\left\{h_{n}\left(q, p ; Q^{*}\right)\right\}_{n=1}^{m}$ such that the system 2.10 is satisfied up to order $b^{m+1}$, i.e.,

$$
\mathcal{H}\left[h^{(m)}\right](q, p)=\mathcal{O}\left(b^{m+1}\right), \mathcal{B}_{0}\left[h^{(m)}\right](q)=\mathcal{O}\left(b^{m+1}\right) \text { and } \mathcal{B}_{1}\left[h^{(m)}\right](q)=0 .
$$

Moreover, the dominant term of the wave height of this approximation of the water wave is of order $b$, i.e,

$$
a^{(m)}=h^{(m)}(0,0 ; b)-h^{(m)}(\pi, 0 ; b)=b\left[h_{1}\left(0,0 ; Q^{*}\right)-h_{1}\left(\pi, 0 ; Q^{*}\right)\right]+\mathcal{O}\left(b^{2}\right) .
$$


This condition indicates the accuracy of approximation of genuine waves in the following sense: The relation $(2.19)$ shows that $h^{(m)}$ is an approximation of a solution up to order $b^{m}$, whereas the relation (2.20) shows that $h^{(m)}$ differs from the laminar solution at order $b$. Thus $h^{(m)}$ is 'closer' to a non-laminar solution.

In Section 3, we present the results for $m=2$. In Section 4 , where we work out the case $m=3$, the situation is different. There we observe that there is no $h^{(3)}(q, p ; b)$ such that both conditions $\mathcal{H}\left[h^{(3)}\right](q, p)=\mathcal{O}\left(b^{4}\right)$ and $\mathcal{B}_{0}\left[h^{(3)}\right](q)=\mathcal{O}\left(b^{4}\right)$ are satisfied. This means that the approximation made on (2.18) is accurate up to $m=2$, and there appears an additional error for $m \geq 3$. Thus, we introduce an auxiliary parameter which allows the control of this additional error. Indeed, we construct the following estimates

$$
\left\|\mathcal{H}\left[h^{(3)}\right](q, p)\right\| \leq \epsilon_{1} b^{3} \text { and }\left\|\mathcal{B}_{0}\left[h^{(3)}\right](q)\right\| \leq \epsilon_{2} b^{3},
$$

for some small $\epsilon_{1} \geq 0$ and $\epsilon_{2} \geq 0$, which depend on this auxiliary parameter.

Moreover, for particular values of the auxiliary parameter we can make each of $\epsilon_{1}$ and $\epsilon_{2}$ arbitrarily small (but not simultaneously), i.e.,

- either, $\mathcal{H}\left[h^{(3)}\right](q, p)=\mathcal{O}\left(b^{4}\right)$,

- or, $\mathcal{B}_{0}\left[h^{(3)}\right](q)=\mathcal{O}\left(b^{4}\right)$.

The selection of the proper value of the auxiliary parameter is discussed in the last parts of Section 4.1 and Section 4.2, as well as in Section 5.2.

\section{Second Order Asymptotics}

The problem of the extension of the asymptotic expansion of the solution of (2.10) takes the following form: with $\lambda_{*}$ defined by $(2.14), H\left(p ; \lambda_{*}\right)$ given by $(2.12)$ and $m(q, p)$ given by (2.15), determine $u(q, p)$ such that the function

$$
h^{*}(q, p ; b)=H\left(p ; \lambda_{*}\right)+b m(q, p)+b^{2} u(q, p),
$$

satisfies the system 2.10 up to the second order, i.e.

$$
\mathcal{H}\left[h^{*}\right](q, p)=\mathcal{O}\left(b^{3}\right), \mathcal{B}_{0}\left[h^{*}\right](q)=\mathcal{O}\left(b^{3}\right) \text { and } \mathcal{B}_{1}\left[h^{*}\right](q)=0 .
$$

Note that, in the absence of stagnation points, the real-analyticity of the wave profile for flows of constant vorticity, established in [10], ensures the existence of a power series expansion. Since the first-order (linear) approximation of the wave profile fails to capture the effects of the underlying current, the issue of obtaining high-order approximations should be explored in this regard.

\subsection{Irrotational flow}

For the purpose of comparison we analyse first the irrotational case $\gamma=0$. This simpler case provides us with the opportunity to explain in more detail the strategy we use throughout this paper to obtain higher order terms of the expansion of the solution. 
In the irrotational case, the laminar solution $H\left(p ; \lambda_{*}\right)$ simplifies to

$$
H\left(p ; \lambda_{*}\right)=\frac{p-p_{0}}{\sqrt{\lambda_{*}}},
$$

the linearised solution $m(q, p)$ becomes

$$
m(q, p)=\sinh \left(\frac{p-p_{0}}{\sqrt{\lambda_{*}}}\right) \cos q,
$$

where $\lambda_{*}>0$ satisfies, in this setting, the dispersion relation

$$
\lambda+g \tanh \left(\frac{p_{0}}{\sqrt{\lambda}}\right)=0 .
$$

Moreover the corresponding value of $Q$ is

$$
Q^{*}=\lambda_{*}-\frac{2 g p_{0}}{\sqrt{\lambda_{*}}} .
$$

We first apply the operator $\mathcal{H}$, defined in $(3.2)$, to the expression $(3.1)$. The outcome will be a polynomial in the $b$ variable, of order up to $b^{6}$. The specific above choices yield the vanishing of the zero and first order of the polynomial; for a detailed discussion of this aspect, we refer to the [11. Our present goal is the vanishing of the $b^{2}$ coefficient of the polynomial.

In particular, using the fact that $H_{q}=0$, we get

$$
\begin{aligned}
\mathcal{H}\left[h^{*}\right]=H_{p p} & +b\left(m_{p p}+H_{p}^{2} m_{q q}\right) \\
& +b^{2}\left[u_{p p}+H_{p}^{2} u_{q q}+H_{p p}-2 H_{p}\left(m_{q} m_{q p}-m_{p} m_{q q}\right)\right]+\mathcal{O}\left(b^{3}\right) .
\end{aligned}
$$

Observing that $(3.3)$ yields

$$
H_{p} \equiv \frac{1}{\sqrt{\lambda_{*}}} \text { and } H_{p p} \equiv 0 \quad \text { for all } \quad p \in\left[p_{0}, 0\right]
$$

and substituting(3.4) in the previous expression for $\mathcal{H}\left[h^{*}\right]$, we get

$$
\mathcal{H}\left[h^{*}\right]=b^{2}\left[u_{p p}+\frac{1}{\lambda_{*}} u_{q q}-\frac{1}{\lambda_{*}} \sinh \left(2 \frac{p-p_{0}}{\sqrt{\lambda_{*}}}\right)\right]+\mathcal{O}\left(b^{3}\right) .
$$

Proceeding similarly for the boundary condition, i.e. for the operator $\mathcal{B}_{0}\left[h^{*}\right]$ in $(3.2)$, yields

$$
\begin{aligned}
\mathcal{B}_{0}\left[h^{*}\right] & =1+(2 g H-Q) H_{p}^{2}+b\left\{2 H_{p}\left[(2 g H-Q) m_{p}+g H_{p} m\right]\right\} \\
& +b^{2}\left[m_{q}^{2}+4 g m H_{p} m_{p}+(2 g H-Q)\left(m_{p}^{2}+2 H_{p} u_{p}\right)+2 g H_{p}^{2} u\right] \\
& +\mathcal{O}\left(b^{3}\right),
\end{aligned}
$$

evaluated at $p=0$. Applying the equalities (3.3), (3.4), (3.6) and (3.7) in the previous expression and using the fact that $(3.5)$ is equivalent to the identities

$$
\cosh \left(\frac{p_{0}}{\sqrt{\lambda_{*}}}\right)=\frac{g}{\sqrt{g^{2}-\lambda_{*}^{2}}} \quad \text { and } \quad \sinh \left(\frac{p_{0}}{\sqrt{\lambda_{*}}}\right)=-\frac{\lambda_{*}}{\sqrt{g^{2}-\lambda_{*}^{2}}}
$$


we get that

$$
\begin{aligned}
\mathcal{B}_{0}\left[h^{*}\right]=-b^{2} 2 \sqrt{\lambda_{*}} & \left\{\left.\left(u_{p}+\frac{g}{\lambda_{*}^{3 / 2}} u\right)\right|_{p=0}\right. \\
& \left.-\frac{1}{4 \sqrt{\lambda_{*}}} \frac{1}{g^{2}-\lambda_{*}^{2}}\left[3 g^{2}+\lambda_{*}^{2}+\left(3 g^{2}-\lambda_{*}^{2}\right) \cos (2 q)\right]\right\} \\
& +\mathcal{O}\left(b^{3}\right) .
\end{aligned}
$$

Consequently, we obtain the constitutive equations for the function $u(q, p)$, even and $2 \pi$ periodic in $q$ variable,

$$
\begin{array}{rlrl}
u_{p p}+\frac{1}{\lambda_{*}} u_{q q} & =\frac{1}{\lambda_{*}} \sinh \left(2 \frac{p-p_{0}}{\sqrt{\lambda_{*}}}\right), & (q, p) \in R, \\
u_{p}-\frac{g}{\lambda_{*}^{3 / 2}} u & =\frac{1}{4 \sqrt{\lambda_{*}}} \frac{1}{g^{2}-\lambda_{*}^{2}}\left[3 g^{2}+\lambda_{*}^{2}+\left(3 g^{2}-\lambda_{*}^{2}\right) \cos (2 q)\right], & p & =0, \\
u & =0, & p & =p_{0} .
\end{array}
$$

In order to solve the above BVP we use separation of variables and we take in consideration the following facts:

- A special solution of the PDE is given by $\frac{1}{4} \sinh \left(2 \frac{p-p_{0}}{\sqrt{\lambda_{*}}}\right)$.

- The function $u(q, p)$ is even and $2 \pi$ periodic.

- We must have $u\left(q, p_{0}\right)=0$.

Therefore, we get the general form

$$
u(q, p)=\frac{1}{4} \sinh \left(\frac{2\left(p-p_{0}\right)}{\sqrt{\lambda_{*}}}\right)+A_{0}\left(p-p_{0}\right)+\sum_{n=2}^{\infty} A_{n} \cos (n q) \sinh \left(\frac{n\left(p-p_{0}\right)}{\sqrt{\lambda_{*}}}\right)
$$

and we have to determine the constants $A_{0}$ and $\left\{A_{n}\right\}_{2}^{\infty}$ such that the Robin Boundary Condition (BC) at $p=0$ is satisfied. Indeed, the separation of variables says that the general solution of the Partial Differential Equation

$$
u_{p p}+\frac{1}{\lambda_{*}} u_{q q}=0
$$

on the rectangle is a bilinear combination of trigonometric functions in $q$ and exponential (hyperbolic) functions in $p$ i.e. $\{\cos (n q), \sin (n q)\}, n \in \mathbb{N}$ and $\exp \left( \pm n p / \sqrt{\lambda_{*}}\right), n \in \mathbb{N}$. This is due to the periodicity in $q 2^{2}$ The evenness in $q$ excludes the dependence on $\sin (n q), n \in \mathbb{N}$ and the condition $u=0$, on $p=p_{0}$ gives exactly the $\sinh \left(n \frac{p-p_{0}}{\sqrt{\lambda_{*}}}\right)$ dependence.

Thus, applying the form $(3.12$ to the second of the equations $(3.11)$, we get

$$
A_{0}=\frac{\lambda_{*}}{4} \frac{3 g^{2}-\lambda_{*}^{2}}{g^{2}-\lambda_{*}^{2}} \frac{1}{g p_{0}+\lambda_{*}^{3 / 2}}, \quad A_{2}=\frac{3 g^{2}-\lambda_{*}^{2}}{8 \lambda_{*}^{2}} \quad \text { and } \quad A_{n}=0, n \geq 3,
$$

with $\lambda_{*}$ given as the solution of (3.5).

\footnotetext{
${ }^{2}$ Once we have obtained $\{\cos (n q), \sin (n q)\}$, separation of variables gives the exponentials $\exp \left( \pm n p / \sqrt{\lambda_{*}}\right)$.
} 


\subsection{Constant Vorticity}

The strategy is conceptually the same for the rotational case when the vorticity $\gamma$ is constant. The first goal is to derive the analogue of the BVP (3.11). In this case, the laminar solution $H(p)$ is given by

$$
H(p)=2 \frac{p-p_{0}}{r(p)+r\left(p_{0}\right)},
$$

where

$$
r(p)=\sqrt{\lambda_{*}-2 \gamma p}
$$

with $\lambda_{*}>0$ satisfying the dispersion relation (2.14). Moreover, the linearised solution $m(q, p)$ is given by 2.15) and rewritten as follows

$$
m(q, p)=\frac{r\left(p_{0}\right)}{r(p)} \sinh (H(p)) \cos q .
$$

The corresponding value of $Q$, using $(2.13)$, is

$$
Q^{*}=\lambda_{*}-\frac{4 g p_{0}}{\sqrt{\lambda_{*}}+\sqrt{\lambda_{*}-2 \gamma p_{0}}} .
$$

Then we evaluate the system 2.10 for $h^{*}$, defined by

$$
h^{*}(q, p ; b)=H(p)+b m(q, p)+b^{2} u(q, p),
$$

with $H(p)$ and $m(q, p)$ given by (3.13) and (3.15), respectively. Demanding the validation of this system up to the second order, i.e.

$$
\mathcal{H}\left[h^{*}\right](q, p)=\mathcal{O}\left(b^{3}\right), \mathcal{B}_{0}\left[h^{*}\right](q)=\mathcal{O}\left(b^{3}\right) \text { and } \mathcal{B}_{1}\left[h^{*}\right](q)=0,
$$

yields the analogue of the BVP (3.11). After tedious but straightforward calculations we get the following form for the BVP, for the even and $2 \pi$-periodic function $u(q, p)$ on the rectangle $R \equiv[-\pi, \pi] \times\left[p_{0}, 0\right]$ :

$$
\begin{aligned}
u_{p p}(q, p)+\frac{1}{r^{2}(p)} u_{q q}(q, p)-\frac{3 \gamma}{r^{2}(p)} u_{p}(q, p) & =f_{0}(p)+f_{2}(p) \cos (2 q), \\
u_{p}(q, 0)-\frac{g}{\lambda_{*}^{3 / 2}} u(q, 0) & =g_{0}+g_{2} \cos (2 q), \\
u\left(q, p_{0}\right) & =0
\end{aligned}
$$

where

$$
\begin{gathered}
f_{0}(p)=-\frac{r^{2}\left(p_{0}\right)}{r^{7}(p)}\left[3 \gamma^{3}-3 \gamma\left(\gamma^{2}+2 r^{2}(p)\right) \cosh \left(2 \frac{r(p)-r\left(p_{0}\right)}{\gamma}\right)\right. \\
\left.+2 r(p)\left(3 \gamma^{2}+2 r^{2}(p)\right) \sinh \left(2 \frac{r(p)-r\left(p_{0}\right)}{\gamma}\right)\right], \\
f_{2}(p)=-\frac{\gamma}{4} \frac{r^{2}\left(p_{0}\right)}{r^{7}(p)}\left[3 \gamma^{2}-2 r^{2}(p)-\left(3 \gamma^{2}+4 r^{2}(p)\right) \cosh \left(2 \frac{r(p)-r\left(p_{0}\right)}{\gamma}\right)\right. \\
\left.+6 \gamma r(p) \sinh \left(2 \frac{r(p)-r\left(p_{0}\right)}{\gamma}\right)\right],
\end{gathered}
$$




$$
\begin{aligned}
& g_{0}=\frac{r^{2}\left(p_{0}\right)}{2 \lambda_{*}} \frac{3 g^{2}+\lambda_{*}^{2}}{g^{2}-\lambda_{*}^{2}-\gamma \sqrt{\lambda_{*}}\left(2 g-\gamma \sqrt{\lambda_{*}}\right)}, \\
& g_{2}=\frac{r^{2}\left(p_{0}\right)}{2 \lambda_{*}} \frac{3 g^{2}-\lambda_{*}^{2}}{g^{2}-\lambda_{*}^{2}-\gamma \sqrt{\lambda_{*}}\left(2 g-\gamma \sqrt{\lambda_{*}}\right)} .
\end{aligned}
$$

Remark 1. The above BVP (3.19) reduces to (3.11) for $\gamma=0$, i.e. the function $f_{2}(p)$ vanishes when $\gamma$ vanishes.

Applying separation of variables to 3.19 , we obtain the following formulation of the above BVP: Determine $u(q, p)$ given by

$$
u(q, p)=u_{0}(p)+\sum_{n=2}^{\infty} u_{n}(p) \cos (n q),
$$

where the functions $u_{n}(p)$ satisfy the following BVPs:

$$
\begin{aligned}
u_{n}^{\prime \prime}(p)-\frac{n^{2}}{r^{2}(p)} u_{n}(p)-\frac{3 \gamma}{r^{2}(p)} u_{n}^{\prime}(p) & =f_{n}(p), \quad p \in\left(p_{0}, 0\right) \\
u_{n}^{\prime}(0)-\frac{g}{\lambda_{*}^{3 / 2}} u_{n}(0) & =g_{n}, \\
u_{n}\left(p_{0}\right) & =0
\end{aligned}
$$

where $f_{0}(p), f_{2}(p), g_{0}$ and $g_{2}$ are defined above. Moreover, $f_{n} \equiv 0$ and $g_{n}=0$, for $n \geq 3$.

Now, we make the following change of variables

$$
u_{n}(p)=\frac{\hat{u}_{n}(r(p))}{r(p)}, \quad \text { with } r(p)=\sqrt{\lambda_{*}+2 \gamma p}
$$

which transforms the above BVPs into the following:

$$
\begin{aligned}
\frac{d^{2}}{d r^{2}} \hat{u}_{n}(r)-\frac{n^{2}}{\gamma^{2}} \hat{u}_{n}(r)=\hat{f}_{n}(r), & r \in\left(r_{0}, \sqrt{\lambda_{*}}\right) \\
\frac{d}{d r} \hat{u}_{n}-\frac{g+\gamma \sqrt{\lambda_{*}} \hat{u}_{n}}{\gamma \lambda_{*}}=\hat{g}_{n}, & r=\sqrt{\lambda_{*}} \\
\hat{u}_{n}=0, & r=r_{0}:=r\left(p_{0}\right)=\sqrt{\lambda_{*}+2 \gamma p_{0}} .
\end{aligned}
$$

Here $\hat{f}_{n}$ and $\hat{g}_{n}$ are defined by

$$
\hat{f}_{n}(r)=\hat{f}_{n}(r(p))=\frac{r^{3}(p)}{\gamma^{2}} f_{n}(p) \quad \text { and } \quad \hat{g}_{n}=-\frac{\lambda_{*}}{\gamma} g_{n}, \quad n \in \mathbb{N} .
$$

The solution basis of the homogeneous differential equations appearing in BVP 3.23 is given by

- $\{1, r\}$ for $n=0$ and

- $\left\{\sinh \left(n \frac{r-r_{0}}{\gamma}\right), \cosh \left(n \frac{r-r_{0}}{\gamma}\right)\right\}$ for $n \geq 1$. 
Using the method of variation of parameters, we obtain the special solutions of the BVP (3.23). In particular, we seek for special solutions of the inhomogeneous problems of the form

- For $n=0$ :

$$
\hat{u}_{0}^{(s)}(r)=\alpha_{0}(r)+\beta_{0}(r) r
$$

under the constraint

$$
\alpha_{0}^{\prime}(r)+\beta_{0}^{\prime}(r) r=0
$$

- For $n \geq 1$ :

$$
\hat{u}_{n}^{(s)}(r)=\alpha_{n}(r) \sinh \left(n \frac{r-r_{0}}{\gamma}\right)+\beta_{n}(r) \cosh \left(n \frac{r-r_{0}}{\gamma}\right)
$$

under the constraint

$$
\alpha_{n}^{\prime}(r) \sinh \left(n \frac{r-r_{0}}{\gamma}\right)+\beta_{n}^{\prime}(r) \cosh \left(n \frac{r-r_{0}}{\gamma}\right)=0 .
$$

The insertion of these forms in the inhomogeneous differential equations appearing in BVP 3.23 yields $\left\{\alpha_{n}, \beta_{n}\right\}, n \in \mathbb{N}$. Consequently, the special solutions read

$$
\hat{u}_{0}^{(s)}(r)=-\gamma r_{0}^{2} \frac{1-\cosh \left(2 \frac{r-r_{0}}{\gamma}\right)}{8 r^{2}}-r_{0}^{2} \frac{\sinh \left(2 \frac{r-r_{0}}{\gamma}\right)}{4 r}
$$

and

$$
\hat{u}_{2}^{(s)}(r)=-\gamma r_{0}^{2} \frac{1-\cosh \left(2 \frac{r-r_{0}}{\gamma}\right)}{8 r^{2}}-r_{0}^{2} \frac{\sinh \left(2 \frac{r-r_{0}}{\gamma}\right)}{4 r} .
$$

The next step is to apply the linear combination

$$
\hat{u}_{n}=\hat{u}_{n}^{(s)}+E_{n} \hat{u}_{n}^{(g)}
$$

to the Robin condition of $(3.23)$ and obtain the specific value for $E_{n}$. One can readily see that $E_{n}=0$ for $n \geq 3$.

Finally, after determining $E_{0}$ and $E_{2}$, we use $(3.22)$ and, by means of $(3.20)$, we obtain the following formula for the second order term of the expansion (3.17):

$$
\begin{aligned}
u(q, p) & =r^{2}\left(p_{0}\right)\left[C_{0} \frac{H(p)}{r(p)}-\gamma \frac{1-\cosh (2 H(p))}{8 r^{3}(p)}+\frac{\sinh (2 H(p))}{4 r^{2}(p)}\right] \\
& +r^{2}\left(p_{0}\right) \cos (2 q)\left[C_{2} \frac{\sinh (2 H(p))}{r(p)}-\gamma \frac{1-\cosh (2 H(p))}{8 r^{3}(p)}+\frac{\sinh (2 H(p))}{4 r^{2}(p)}\right],
\end{aligned}
$$

where

$$
C_{0}=\frac{\sqrt{\lambda_{*}}\left[3 g\left(g-\gamma \sqrt{\lambda_{*}}\right)+\lambda_{*}\left(\gamma^{2}-\lambda_{*}\right)\right]\left(r\left(p_{0}\right)+\sqrt{\lambda_{*}}\right)}{4\left[\left(g-\gamma \sqrt{\lambda_{*}}\right)^{2}-\lambda_{*}^{2}\right]\left[2 g p_{0}+\lambda_{*} r\left(p_{0}\right)+\sqrt{\lambda_{*}} r^{2}\left(p_{0}\right)\right]}
$$


and

$$
C_{2}=\frac{3 g\left(g-\gamma \sqrt{\lambda_{*}}\right)+\lambda_{*}\left(\gamma^{2}-3 \lambda_{*}\right)}{8 \lambda_{*}^{5 / 2}} .
$$

Therefore, the function $h^{*}$ given by (3.17) satisfies the system (3.18). We note that for the value $\gamma=0$, the expression (3.24) takes the form (3.12), meaning that we obtain the irrotational case as a special case of the constant vorticity formulation.

\section{Third Order}

With a similar methodology as that used to derive approximations of second order, one can derive approximation of third order. The results are provided below; the reader can easily verify them with some Computer Algebra Software.

In particular, we use the formula

$$
\tilde{h}(q, p ; b)=H(p)+b m(q, p)+b^{2} u(q, p)+b^{3} w(q, p),
$$

with $H, m$ and $u$ given in the previous section and $\lambda_{*}$ given by 2.14). Then our aim is to determine $w$ so that $\tilde{h}$ satisfies the system (2.10) up to the third order, i.e.

$$
\mathcal{H}[\tilde{h}](q, p)=\mathcal{O}\left(b^{4}\right), \mathcal{B}_{0}[\tilde{h}](q)=\mathcal{O}\left(b^{4}\right) \text { and } \mathcal{B}_{1}[\tilde{h}](q)=0 .
$$

However, there is no such $\tilde{h}(q, p ; b)$. Thus, instead, we introduce an auxiliary parameter $\tilde{B}$ (for the irrotational case we denote it by $B$ ) which allows the control of this additional error estimate. Indeed, we construct the following estimates

$$
\left\|\mathcal{H}\left[h^{(3)}\right](q, p)\right\| \leq \epsilon_{1} b^{3} \text { and }\left\|\mathcal{B}_{0}\left[h^{(3)}\right](q)\right\| \leq \epsilon_{2} b^{3},
$$

for some small $\epsilon_{1} \geq 0$ and $\epsilon_{2} \geq 0$, which depend on this auxiliary parameter.

\subsection{Irrotational case}

Following the organisation of the previous section we first discuss the case that $\gamma=0$. Therefore, if we apply (4.1) to the system (2.10), we get a polynomial on $b$ of finite order (in particular of order $\left.b^{9}\right)$. The requirement that the coefficient of the $b^{3}$ term vanishes yields the following BVP for the even and $2 \pi$-periodic (in the $q$ variable) function $w(q, p)$ :

$$
\begin{array}{rlrl}
w_{p p}+\frac{1}{\lambda_{*}} w_{q q} & =f_{1}(p) \cos q+f_{3}(p) \cos (3 q), & (q, p) \in R, \\
w_{p}-\frac{g}{\lambda_{*}^{3 / 2}} w & =g_{1} \cos q+g_{3} \cos (3 q), & p & =0, \\
w & =0, & p & =p_{0},
\end{array}
$$

where $f_{1}(p)$ and $f_{3}(p)$ are known functions (bilinear combinations of hyperbolic functions and polynomials) and $g_{1}$ and $g_{3}$ are known constants, dependent on the parameters $g, p_{0}$ and $\lambda_{*}$. We use the fact that this problem has the same general solution (for the homogeneous case) 
with the problem (3.11) and we determine its special solutions by the method of variation of parameters. Then the expansion

$$
\tilde{h}(q, p ; b)=H(p)+b m(q, p)+b^{2} u(q, p)+b^{3} \tilde{w}(q, p),
$$

with $\tilde{w}(q, p)$ given the following formula

$$
\begin{aligned}
\tilde{w}(q, p) & =\left[B_{1}\left(p-p_{0}\right) \cosh \left(\frac{p-p_{0}}{\sqrt{\lambda_{*}}}\right)+B_{2} \sinh \left(3 \frac{p-p_{0}}{\sqrt{\lambda_{*}}}\right)\right] \cos q \\
& +\left[D_{1} \sinh \left(\frac{p-p_{0}}{\sqrt{\lambda_{*}}}\right)+D_{2} \sinh \left(3 \frac{p-p_{0}}{\sqrt{\lambda_{*}}}\right)\right] \cos (3 q),
\end{aligned}
$$

with

$$
\begin{gathered}
B_{1}=\frac{\lambda_{*}}{4} \frac{3 g^{2}-\lambda_{*}^{2}}{g^{2}-\lambda_{*}^{2}} \frac{1}{g p_{0}+\lambda_{*}^{3 / 2}}, \quad B_{2}=\frac{9 g^{2}}{32 \lambda_{*}^{2}}, \\
D_{1}=-\frac{3 g^{2}-\lambda_{*}^{2}}{32 \lambda_{*}^{2}} \quad \text { and } \quad D_{2}=\frac{9 g^{2}}{32 \lambda_{*}^{2}}+\frac{3 g^{2}-\lambda_{*}^{2}}{32 \lambda_{*}^{2}},
\end{gathered}
$$

satisfies the conditions

$$
\mathcal{H}[\tilde{h}](q, p)=\mathcal{O}\left(b^{4}\right) \text { and } \mathcal{B}_{1}[\tilde{h}](q)=0 .
$$

For the Robin boundary condition we take

$$
\mathcal{B}_{0}[\tilde{h}](q)=-B_{0} \cos q b^{3}+\mathcal{O}\left(b^{4}\right),
$$

with the constant $B_{0}$ given explicitly by the expression

$$
B_{0}=\frac{1}{2 \lambda_{*}} \frac{1}{\left(g^{2}-\lambda_{*}^{2}\right)^{3 / 2}}\left[\frac{\lambda_{*} p_{0}\left(3 g^{2}-\lambda_{*}^{2}\right)^{2}}{g p_{0}+\lambda_{*}^{3 / 2}}-\frac{g\left(3 g^{2}+\lambda_{*}^{2}\right)^{2}}{2 \lambda_{*}}\right] .
$$

Thus, we propose the following formulation

$$
w(q, p)=\tilde{w}(q, p)-B\left(p-p_{0}\right) \cos q,
$$

for some constant $B$. Then the condition (4.2) changes accordingly. In particular, (4.5) becomes

$$
\mathcal{H}[\tilde{h}](q, p)=B \frac{p-p_{0}}{\lambda_{*}} \cos q b^{3}+\mathcal{O}\left(b^{4}\right)
$$

and the Robin boundary condition takes the following form:

$$
\mathcal{B}_{0}[\tilde{h}](q)=\left[\frac{2}{\lambda_{*}}\left(g p_{0}+\lambda_{*}^{3 / 2}\right) B-B_{0}\right] \cos q b^{3}+\mathcal{O}\left(b^{4}\right) .
$$

Therefore we can choose some appropriate value of $B$ in order to minimize the errors observed in the above conditions. For example,

- $B=0$ ensures $\mathcal{H}[\tilde{h}]=\mathcal{O}\left(b^{4}\right)$.

- $B=B_{0} \frac{\lambda_{*}}{2} \frac{1}{g p_{0}+\lambda_{*}^{3 / 2}}$ ensures $\mathcal{B}_{0}[\tilde{h}]=\mathcal{O}\left(b^{4}\right)$ 
We observe the magnitude of the error of the $b^{3}$ term, for the operators $\mathcal{H}$ and $\mathcal{B}_{0}$, to be

$$
\mathcal{E}_{d}:=B \frac{-p_{0}}{\lambda_{*}} \geq 0
$$

and

$$
\mathcal{E}_{c}:=B_{0}-\frac{2}{\lambda_{*}}\left(g p_{0}+\lambda_{*}^{3 / 2}\right) B \geq 0
$$

respectively, with

$$
0 \leq B \leq B_{0} \frac{\lambda_{*}}{2} \frac{1}{g p_{0}+\lambda_{*}^{3 / 2}}
$$

\subsection{Constant vorticity}

While the situation is similar for this case, it is, however, much more complicated. Indeed, we apply the expansion of the form (4.1), with $H, m$ and $u$ given by (3.13), (3.15) and (3.24), respectively, to the system (4.2) and we get the following BVP on the rectangular $R$, for the even and periodic function $w(q, p)$ :

$$
\begin{aligned}
w_{p p}(q, p)+\frac{1}{r^{2}(p)} w_{q q}(q, p)-\frac{3 \gamma}{r^{2}(p)} w_{p}(q, p) & =f_{1}(p) \cos q+f_{3}(p) \cos (3 q), \\
w_{p}(q, 0)-\frac{g}{\lambda_{*}^{3 / 2}} w(q, 0) & =g_{1} \cos q+g_{3} \cos (3 q), \\
w\left(q, p_{0}\right) & =0,
\end{aligned}
$$

where

- $f_{1}(p)$ and $f_{3}(p)$ are known functions explicitly dependent on $r(p)$,

- $g_{1}$ and $g_{3}$ are known constants explicitly dependent on the parameters $\gamma, \lambda_{*}, p_{0}$ and $g$,

for which we avoid to write the complete expressions for matters of brevity. Proceeding as in Section 3.2, we reduce the solution of the above BVP to the following formulation: Determine $w(q, p)$, of the form

$$
w(q, p)=\sum_{n=0}^{\infty} w_{n}(p) \cos (n q),
$$

with $w_{n}(p)$ given by

$$
w_{n}(p)=\frac{\hat{w}_{n}(r(p))}{r(p)} \quad \text { with } r(p)=\sqrt{\lambda_{*}-2 \gamma p}
$$

and where $\hat{w}_{n}(r)$ are the solutions of the BVPs

$$
\begin{aligned}
\frac{d^{2}}{d r^{2}} \hat{w}_{n}(r)-\frac{n^{2}}{\gamma^{2}} \hat{w}_{n}(r)=\hat{f}_{n}(r), & r \in\left(r_{0}, \sqrt{\lambda_{*}}\right) \\
\frac{d}{d r} \hat{w}_{n}+\frac{g-\gamma \sqrt{\lambda_{*}}}{\gamma \lambda_{*}} \hat{w}_{n}=\hat{g}_{n}, & r=\sqrt{\lambda_{*}} \\
\hat{w}_{n}=0, & r=r_{0}=\sqrt{\lambda_{*}-2 \gamma p_{0}},
\end{aligned}
$$

for some known functions $\hat{f}_{n}(r)$ and constants $\hat{g}_{n}$. 
Remark 2. The functions $\hat{f}_{n}(r)$ are bilinear combinations of hyperbolic functions and polynomials in $1 / r$. The constants $\hat{g}_{n}$ are dependent on $\gamma, g, \lambda_{*}$ and $r_{0}$.

Eventually, using the arguments of the previous subsection we derive the following formula for $w(q, p)$ :

$$
w(q, p)=\tilde{w}(q, p)-\tilde{B} r^{2}\left(p_{0}\right) \frac{H(p)}{r(p)} \cos q,
$$

for some constant $\tilde{B}$ and $\tilde{w}(q, p)$ given by

$$
\begin{aligned}
\tilde{w}(q, p)= & {\left[\tilde{A}_{1}(p) \sinh (H(p))+\tilde{A}_{2}(p) \sinh (3 H(p))\right.} \\
& \left.+\tilde{B}_{1}(p) \cosh (H(p))+\tilde{B}_{2}(p) \cosh (3 H(p))\right] \cos q \\
+ & {\left[\tilde{A}_{3}(p) \sinh (H(p))+\tilde{A}_{4}(p) \sinh (3 H(p))\right.} \\
& \left.+\tilde{B}_{3}(p) \cosh (H(p))+\tilde{B}_{4}(p) \cosh (3 H(p))\right] \cos (3 q),
\end{aligned}
$$

where $\left\{\tilde{A}_{j}(p), \tilde{B}_{j}(p)\right\}_{j=1}^{4}$ are functions of the form

$$
\frac{1}{r^{5}(p)} \sum_{n=0}^{4} a_{j, n} r^{n}(p) \quad \text { and } \quad \frac{1}{r^{5}(p)} \sum_{n=0}^{4} b_{j, n} r^{n}(p),
$$

respectively. Here

$$
\begin{aligned}
& a_{1,0}=- \frac{9}{32} \gamma^{2} r_{0}^{3}, \quad a_{1,1}=0, \\
& a_{1,2}=-\left\{r _ { 0 } ^ { 3 } \left(3 g\left(g-\gamma \sqrt{\lambda_{*}}-\lambda_{*}\right) \sqrt{\lambda_{*}}\left(g-\gamma \sqrt{\lambda_{*}}+\lambda_{*}\right),\right.\right. \\
&\left.\left.+r_{0}\left(-3 g^{3}-15 g^{2} \gamma \sqrt{\lambda_{*}}-5 \gamma\left(\gamma^{2}-\lambda_{*}\right) \lambda_{*}^{3 / 2}+3 g \lambda_{*}\left(5 \gamma^{2}+\lambda_{*}\right)\right)\right)\right\} / \\
&\left\{32\left(-r_{0}\left(g-\gamma \sqrt{\lambda_{*}}\right)+g \sqrt{\lambda_{*}}\right)\left(g^{2}-2 g \gamma \sqrt{\lambda_{*}}+\left(\gamma^{2}-\lambda_{*}\right) \lambda_{*}\right)\right\}, \\
& a_{1,3}=- \frac{r_{0}^{3}\left(3 g^{2}-3 g \gamma \sqrt{\lambda_{*}}+\left(\gamma^{2}-3 \lambda_{*}\right) \lambda_{*}\right)}{32 \lambda_{*}^{5 / 2}}, \quad a_{1,4}=0, \\
& b_{1,0}=0, \quad b_{1,1}=-\frac{9 \gamma r_{0}^{3}}{32}, \quad b_{1,2}=-\frac{\gamma r_{0}^{3}\left(3 g^{2}-3 g \gamma \sqrt{\lambda_{*}}+\left(\gamma^{2}-3 \lambda_{*}\right) \lambda_{*}\right)}{32 \lambda_{*}^{5 / 2}}, \\
& b_{1,3}=\frac{r_{0}^{4} \sqrt{\lambda_{*}}\left(-3 g^{2}+3 g \gamma \sqrt{\lambda_{*}}+\lambda_{*}\left(-\gamma^{2}+\lambda_{*}\right)\right)}{4\left(g r_{0}-\left(g+\gamma r_{0}\right) \sqrt{\lambda_{*}}\right)\left(g^{2}-2 g \gamma \sqrt{\lambda_{*}}+\left(\gamma^{2}-\lambda_{*}\right) \lambda_{*}\right)}, \\
& b_{1,4}=\frac{r_{0}^{3} \sqrt{\lambda_{*}}\left(3 g^{2}-3 g \gamma \sqrt{\lambda_{*}}+\left(\gamma^{2}-\lambda_{*}\right) \lambda_{*}\right)}{4\left(g r_{0}-\left(g+\gamma r_{0}\right) \sqrt{\lambda_{*}}\right)\left(g-\gamma \sqrt{\lambda_{*}}-\lambda_{*}\right)\left(g-\gamma \sqrt{\lambda_{*}}+\lambda_{*}\right)},
\end{aligned}
$$




$$
\begin{aligned}
& a_{2,0}=\frac{3}{32} \gamma^{2} r_{0}^{3}, \quad a_{2,1}=0, \quad a_{2,2}=\frac{9 r_{0}^{3}}{32}, \\
& a_{2,3}=\frac{3 r_{0}^{3}\left(3 g^{2}-3 g \gamma \sqrt{\lambda_{*}}+\left(\gamma^{2}-3 \lambda_{*}\right) \lambda_{*}\right)}{32 \lambda_{*}^{5 / 2}}, \quad a_{2,4}=0, \\
& b_{2,0}=0, \quad b_{2,1}=\frac{9}{32} \gamma r_{0}^{3}, \\
& b_{2,2}=\frac{\gamma r_{0}^{3}\left(3 g^{2}-3 g \gamma \sqrt{\lambda_{*}}+\left(\gamma^{2}-3 \lambda_{*}\right) \lambda_{*}\right)}{32 \lambda_{*}^{5 / 2}}, \quad b_{2,3}=b_{2,4}=0 \text {, } \\
& a_{3,0}=-\frac{3}{32} \gamma^{2} r_{0}^{3}, \quad a_{3,1}=0, \quad a_{3,2}=-\frac{r_{0}^{3}}{32}, \\
& a_{3,3}=-\frac{r_{0}^{3}\left(3 g^{2}-3 g \gamma \sqrt{\lambda_{*}}+\left(\gamma^{2}-3 \lambda_{*}\right) \lambda_{*}\right)}{32 \lambda_{*}^{5 / 2}}, \quad a_{3,4}=0, \\
& b_{3,0}=0, \quad b_{3,1}=-\frac{3 \gamma r_{0}^{3}}{32}, \\
& b_{3,2}=-\frac{\gamma r_{0}^{3}\left(3 g^{2}-3 g \gamma \sqrt{\lambda_{*}}+\left(\gamma^{2}-3 \lambda_{*}\right) \lambda_{*}\right)}{32 \lambda_{*}^{5 / 2}}, \quad b_{3,3}=b_{3,4}=0 \text {, } \\
& a_{4,0}=\frac{1}{32} \gamma^{2} r_{0}^{3}, \quad a_{4,1}=0, \quad a_{4,2}=\frac{3 r_{0}^{3}}{32}, \\
& a_{4,3}=\frac{3 r_{0}^{3}\left(3 g^{2}-3 g \gamma \sqrt{\lambda_{*}}+\left(\gamma^{2}-3 \lambda_{*}\right) \lambda_{*}\right)}{32 \lambda_{*}^{5 / 2}}, \\
& a_{4,4}=\left\{r _ { 0 } ^ { 3 } \left(9 g^{5}-27 g^{4} \gamma \sqrt{\lambda_{*}}+11 g^{3}\left(3 \gamma^{2}-2 \lambda_{*}\right) \lambda_{*}-g^{2} \gamma\left(21 \gamma^{2}-50 \lambda_{*}\right) \lambda_{*}^{3 / 2}\right.\right. \\
& \left.\left.+g \lambda_{*}^{2}\left(7 \gamma^{4}-35 \gamma^{2} \lambda_{*}+13 \lambda_{*}^{2}\right)-\gamma \lambda_{*}^{5 / 2}\left(\gamma^{4}-9 \gamma^{2} \lambda_{*}+15 \lambda_{*}^{2}\right)\right)\right\} / \\
& \left\{64\left(g-\gamma \sqrt{\lambda_{*}}\right) \lambda_{*}^{5}\right\} \\
& b_{4,0}=0, \quad b_{4,1}=\frac{3}{32} \gamma r_{0}^{3}, \\
& b_{4,2}=\frac{\gamma r_{0}^{3}\left(3 g^{2}-3 g \gamma \sqrt{\lambda_{*}}+\left(\gamma^{2}-3 \lambda_{*}\right) \lambda_{*}\right)}{32 \lambda_{*}^{5 / 2}}, \quad b_{4,3}=b_{4,4}=0,
\end{aligned}
$$

where we used the abbreviation $r\left(p_{0}\right)=r_{0}$. Consequently, the conditions 4.2 take on the following form:

$$
\mathcal{H}[\tilde{h}](q, p)=\tilde{B} r^{2}\left(p_{0}\right) \frac{H(p)}{r^{3}(p)} \cos q b^{3}+\mathcal{O}\left(b^{4}\right)
$$

and

$$
\mathcal{B}_{0}[\tilde{h}](q)=\left[2 r^{2}\left(p_{0}\right) \frac{2 g p_{0}+r\left(p_{0}\right) \sqrt{\lambda_{*}}\left(r\left(p_{0}\right)+\sqrt{\lambda_{*}}\right)}{\lambda_{*}^{3 / 2}\left(r\left(p_{0}\right)+\sqrt{\lambda_{*}}\right)} \tilde{B}-\tilde{B}_{0}\right] \cos q b^{3}+\mathcal{O}\left(b^{4}\right),
$$

with

$$
\tilde{B}_{0}=D \frac{\sum_{n=0}^{5} c_{n} \gamma^{n}}{g^{2}-\lambda_{*}^{2}-\gamma \sqrt{\lambda_{*}}\left(2 g-\gamma \sqrt{\lambda_{*}}\right)}
$$


where

$$
D=-\frac{1}{4 \lambda_{*}^{7 / 2}} \frac{r\left(p_{0}\right)+\sqrt{\lambda_{*}}}{2 g p_{0}+r\left(p_{0}\right) \sqrt{\lambda_{*}}\left(r\left(p_{0}\right)+\sqrt{\lambda_{*}}\right)}
$$

and

$$
\begin{gathered}
c_{0}=3 g \sqrt{\lambda_{*}}\left(9 g^{4}-14 g^{2} \lambda_{*}^{2}+5 \lambda_{*}^{4}\right)-4 g \sqrt{\lambda_{*}} r_{0}\left(9 g^{4}-9 g^{2} \lambda_{*}^{2}+4 \lambda_{*}^{4}\right) \\
-\frac{2 p_{0}}{\sqrt{\lambda_{*}}+r_{0}}\left(9 g^{6}-12 g^{4} \lambda_{*}^{2}+13 g^{2} \lambda_{*}^{4}-2 \lambda_{*}^{6}\right), \\
c_{1}=-\lambda_{*}^{3 / 2}\left(33 g^{4}-39 g^{2} \lambda_{*}^{2}+4 \lambda_{*}^{4}\right)-r_{0}\left(-60 g^{4} \lambda_{*}+45 g^{2} \lambda_{*}^{3}-7 \lambda_{*}^{5}\right), \\
c_{2}=-3\left(-7 g^{3} \lambda_{*}^{2}+5 g \lambda_{*}^{4}+2 g r_{0} \lambda_{*}^{3 / 2}\left(9 g^{2}-4 \lambda_{*}^{2}\right)\right), \\
c_{3}=-\lambda_{*}^{5 / 2}\left(7 g^{2}-2 \lambda_{*}^{2}\right)-r_{0}\left(-28 g^{2} \lambda_{*}^{2}+5 \lambda_{*}^{4}\right), \\
c_{4}=-8 g r_{0} \lambda_{*}^{5 / 2}+g \lambda_{*}^{3}, \quad c_{5}=r_{0} \lambda_{*}^{3} .
\end{gathered}
$$

Similarly to the previous section we observe that the magnitude of the error of the $b^{3}$ term, for the operators $\mathcal{H}$ and $\mathcal{B}_{0}$, to be

$$
\mathcal{E}_{d}:=\tilde{B} \frac{H\left(p_{0}\right)}{r\left(p_{0}\right)} \geq 0
$$

and

$$
\mathcal{E}_{c}:=\tilde{B}_{0}-2 r^{2}\left(p_{0}\right) \frac{2 g p_{0}+r\left(p_{0}\right) \sqrt{\lambda_{*}}\left(r\left(p_{0}\right)+\sqrt{\lambda_{*}}\right)}{\lambda_{*}^{3 / 2}\left(r\left(p_{0}\right)+\sqrt{\lambda_{*}}\right)} \tilde{B} \geq 0,
$$

respectively, with

$$
0 \leq \tilde{B} \leq \tilde{B}_{0} \frac{\lambda_{*}^{3 / 2}}{2 r^{2}\left(p_{0}\right)} \frac{r\left(p_{0}\right)+\sqrt{\lambda_{*}}}{2 g p_{0}+r\left(p_{0}\right) \sqrt{\lambda_{*}}\left(r\left(p_{0}\right)+\sqrt{\lambda_{*}}\right)} .
$$

Remark 3. One can see that the case $\gamma=0$, equivalent to $r(p)=\sqrt{\lambda_{*}}$ for all $p \in\left[p_{0}, 0\right]$, in the above expressions yields the formulae of the previous subsection.

\section{Illustration of the solutions}

Considering the figures depicted in this section, we recall that the value of the gravitational constant of acceleration is $g=9.8$ and we fix the relative mass flux $p_{0}=-2$. This section is organised as follows. In the first subsection we illustrate the results for the second order asymptotic expansion. In the second subsection we present the analogue results for the third order expansions. In the third subsection we present some figures that compare the obtained second order approximation results against existing results i.e. first order asymptotic expansions. Finally, in the fourth subsection we compare the results between the second and the third order approximations.

The illustrations below are given for several values of the vorticity $\gamma$, as indicated in each figure. Moreover, each streamline is defined by $\left[p=\right.$ constant], for constants ranging from $p_{0}$ to 0 . We note that for the wave height $h$ and the velocity field $v$, we have that: 
- $p=p_{0}$ gives $h=0$ and $v=0$.

- $h$ is an increasing function with respect to $p$.

- $v$ is an increasing function with respect to $p$, for $q>0$, and a decreasing function with respect to $p$, for $q<0$.

whereas, for the pressure $P$ :

- $P(q, 0)=P_{a t m}(q)=P_{a t m}$.

- $P$ is a decreasing function with respect to $p$.

Let us recall the definition of the wave height as the maximal oscillation of the free boundary, $h(0,0)-h(\pi, 0)$, with the understanding the the wave crest is located at $q=0$. Moreover, we recall from [12] that:

- The velocity field is given by $(c-u, v)=\left(\frac{1}{h_{p}},-\frac{h_{q}}{h_{p}}\right)$.

- The water pressure is given by

$$
P=P_{a t m}-\frac{1+h_{q}^{2}}{2 h_{p}^{2}}-g h-\gamma p+\frac{Q}{2}
$$

for some constant $Q$.

\subsection{Second order}

In this subsection we depict the results that we obtain from the second order asymptotic expansion of the solution $h(q, p)$, given in (3.17). Concerning the other parameters, we remind that $\lambda_{*}$ varies with the vorticity according to the dispersion relation (2.14).

Firstly, for each value of the constant vorticity $\gamma$, we have to fix the value of $b$ by allowing a small error on condition 3.18 , i.e.

$$
\max _{b}\left\{\|\mathcal{H}\|_{2},\left\|\mathcal{B}_{0}\right\|_{2}\right\}=\epsilon \text { for some small } \epsilon>0 .
$$

In Figure 3 we show how $b$ varies for different values of vorticity. The value $b$ is increasing when $\gamma \in[-4.5,-1.5]$, with $\|\mathcal{H}\|_{2}$ dominant in the above definition. On the contrary, the value of $b$ is decreasing when $\gamma \in[-1.5,3]$, with $\left\|\mathcal{B}_{0}\right\|_{2}$ dominant in the above definition. 


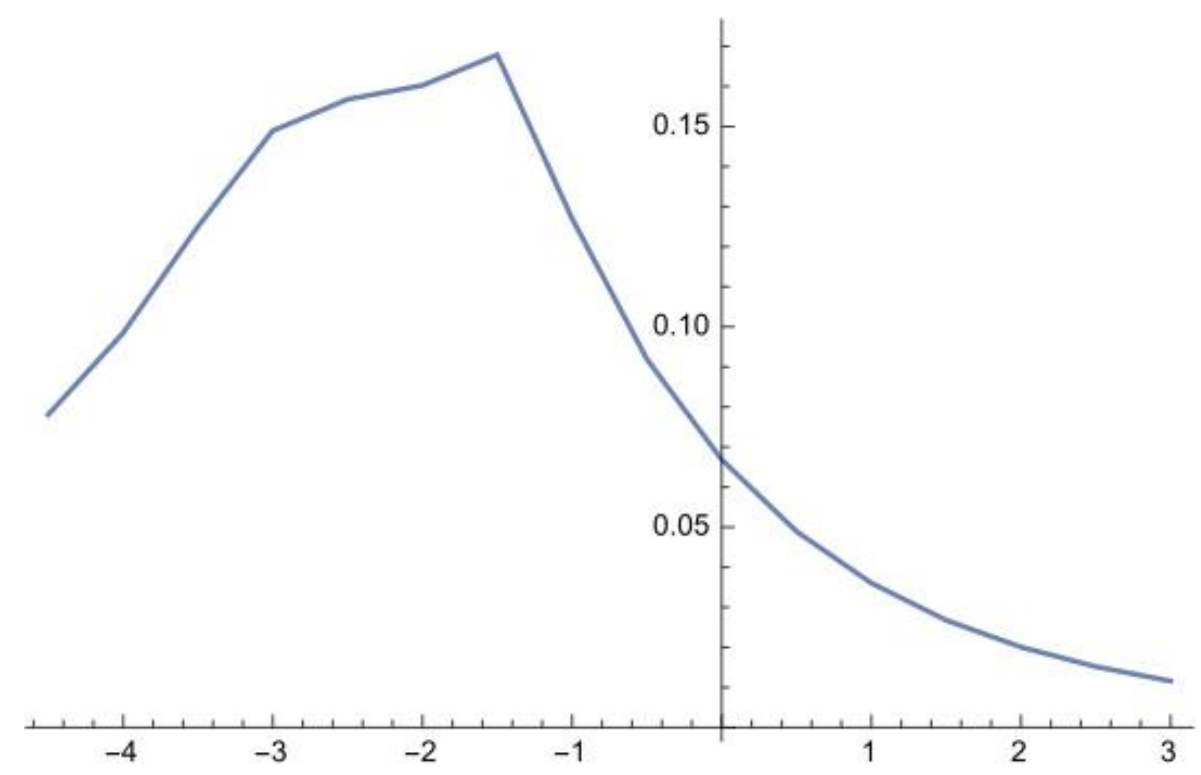

Figure 3: The value of $b$ for different values of vorticity, varying from $\gamma=-4.5$ to $\gamma=3$.

\subsubsection{Wave profiles}

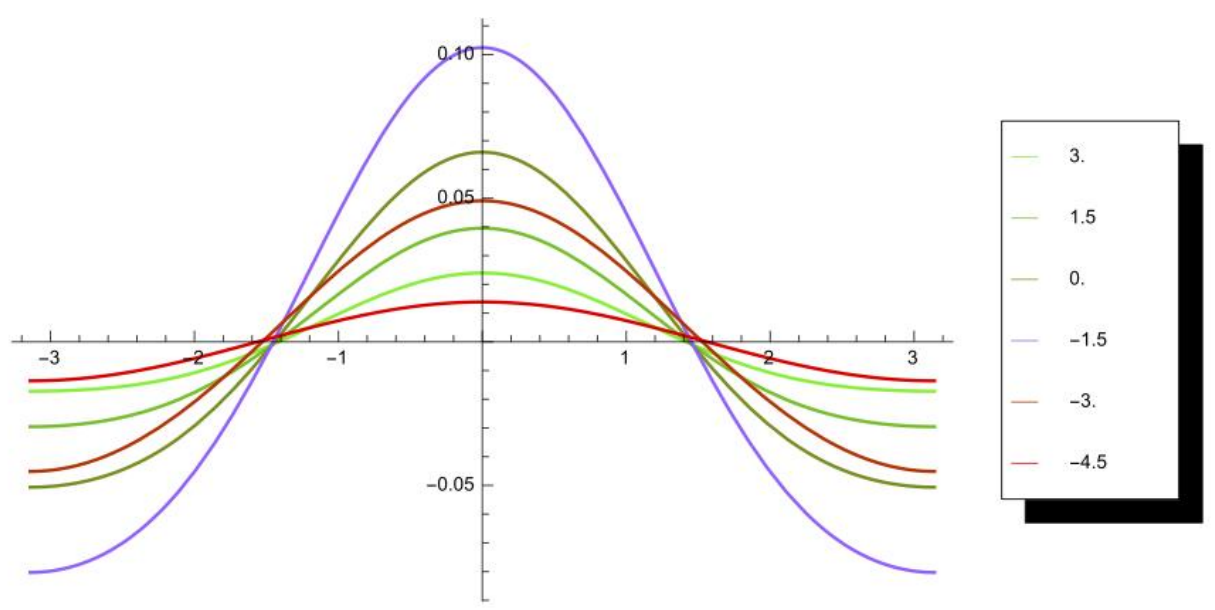

Figure 4: The free surface for different values of vorticity, varying from $\gamma=-4.5$ to $\gamma=3$. Here the wave profile $\eta(x)$ is illustrated, which is oscillating around $y=0$. 


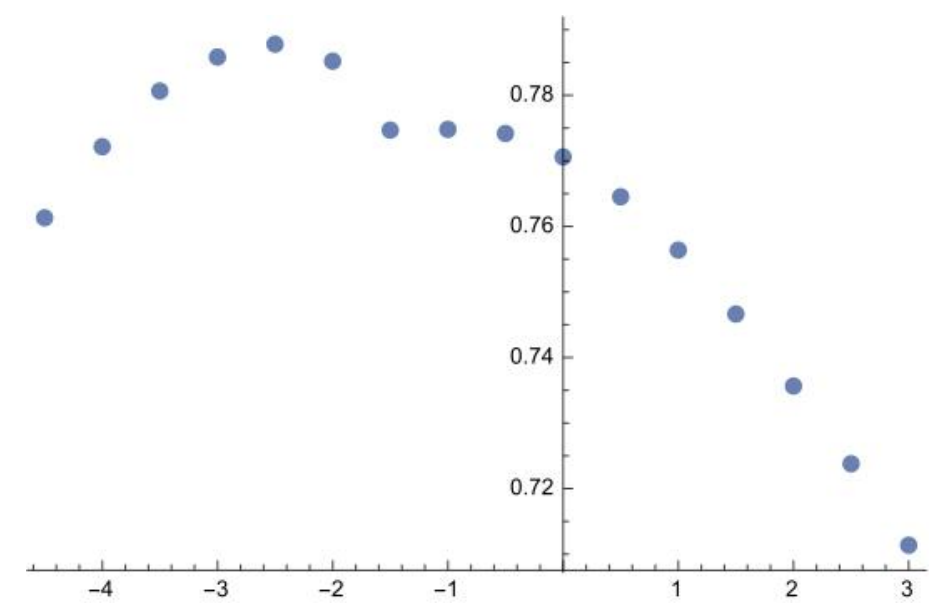

Figure 5: The mean depth for different values of vorticity, varying from $\gamma=-4.5$ to $\gamma=3$.

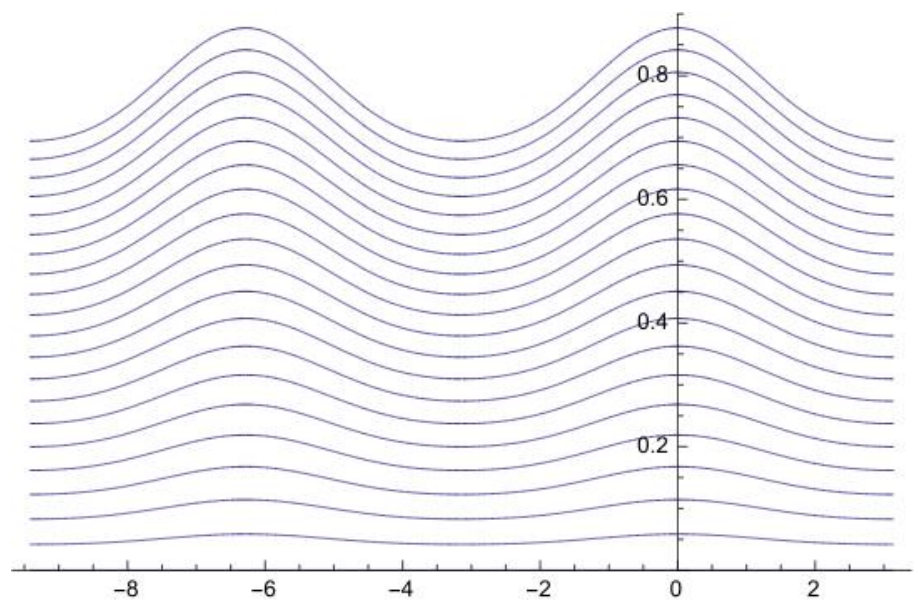

Figure 6: The height of the water along streamlines for $\gamma=-1.5$, over two wavelengths.

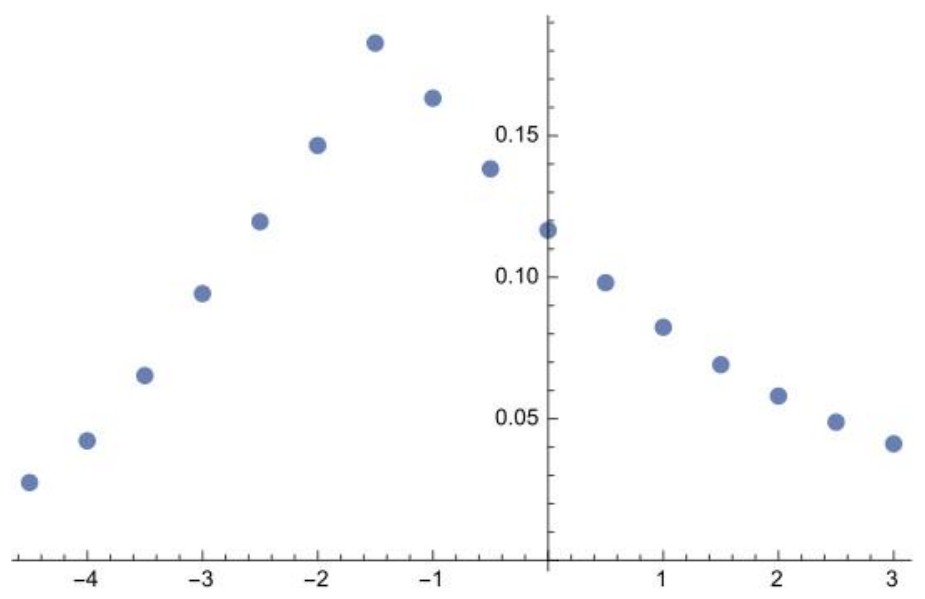

Figure 7: The wave height for different values of vorticity, varying from $\gamma=-4.5$ to $\gamma=3$. 


\subsubsection{Velocities}

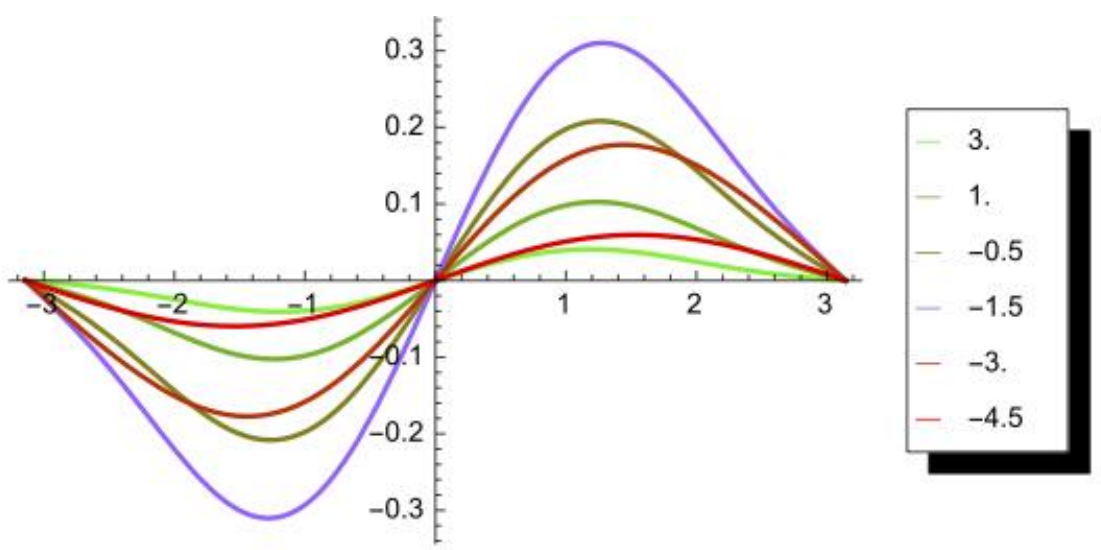

Figure 8: The vertical velocity $v$ on free surface for different values of vorticity, varying from $\gamma=-4.5$ to $\gamma=3$.

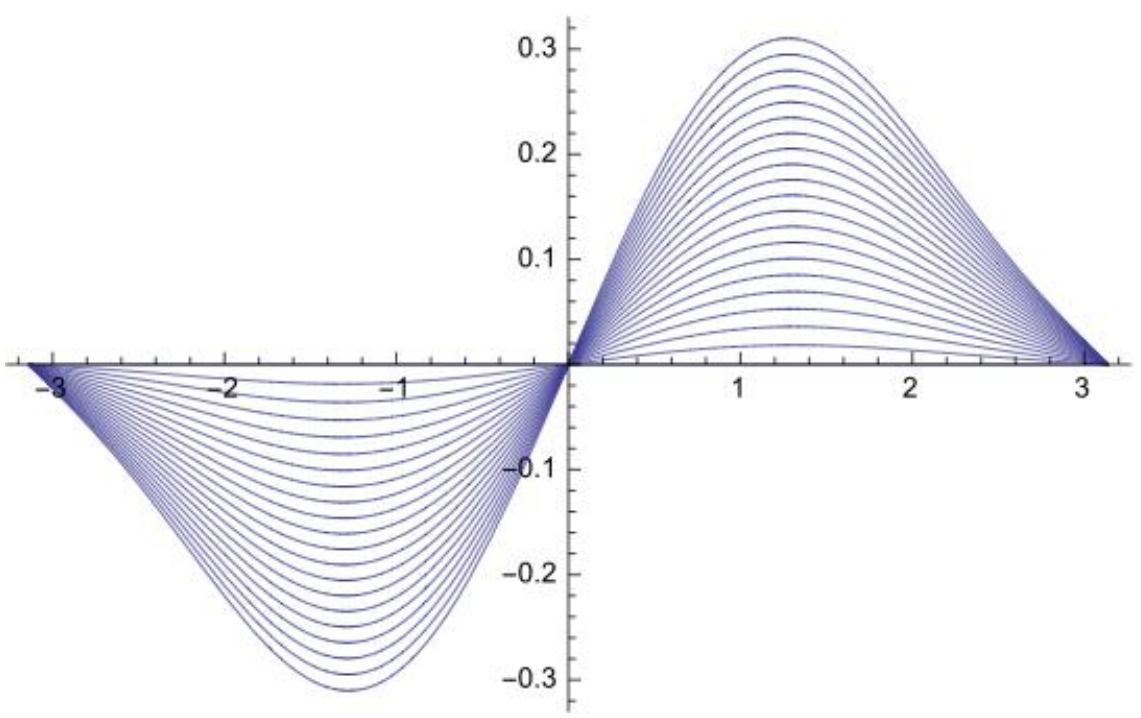

Figure 9: The vertical velocity $v$ of the flow along streamlines for $\gamma=-1.5$. 


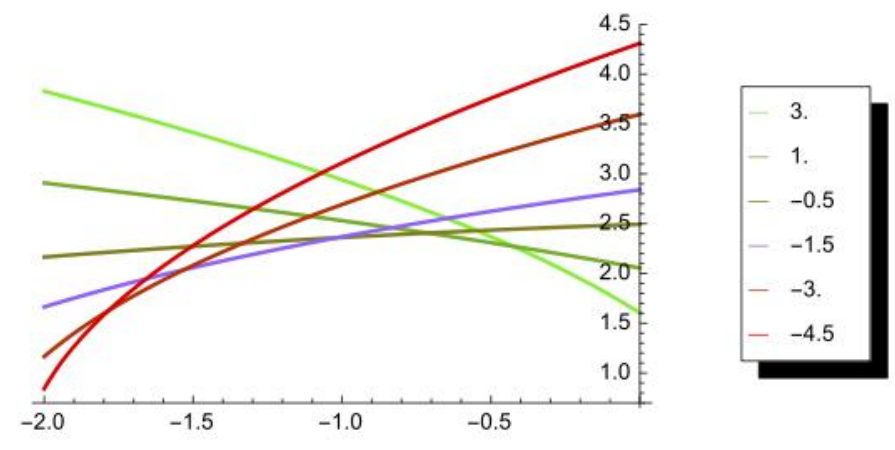

Figure 10: The horizontal velocity $c-u$ on the straight line below the crest, i.e. $q=0$ and $p \in\left[p_{0}, 0\right]$, for different values of vorticity, varying from $\gamma=-4.5$ to $\gamma=3$.

\subsubsection{Pressure}

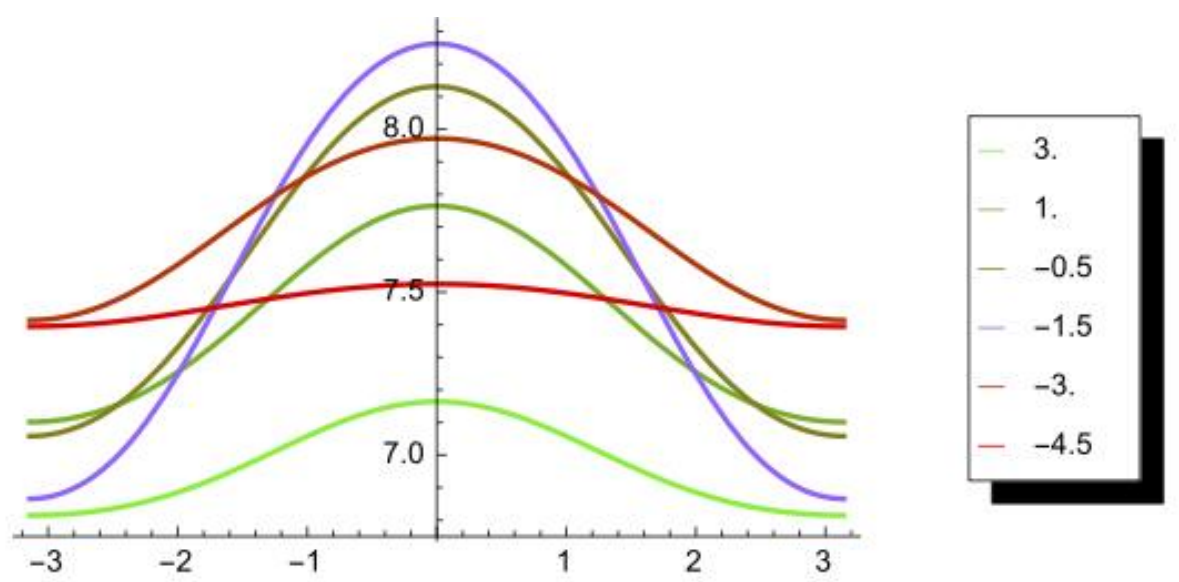

Figure 11: The water pressure on the bottom, $p=p_{0}$, for different values of vorticity, varying from $\gamma=-4.5$ to $\gamma=3$. 


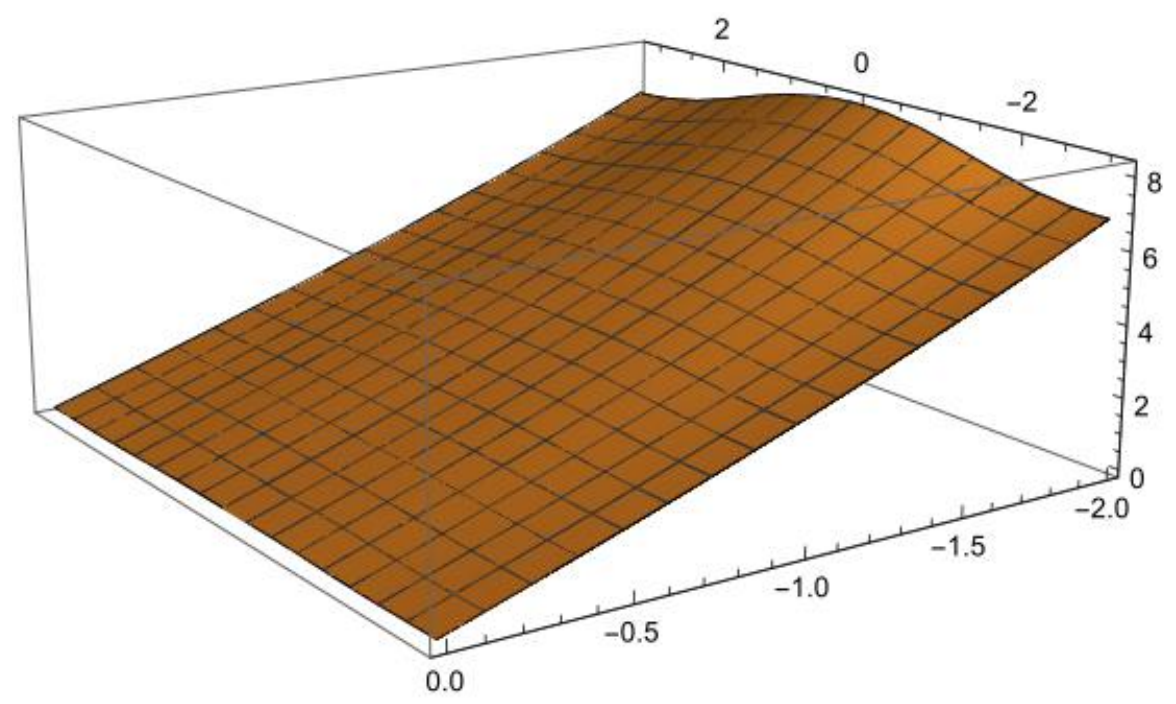

Figure 12: The water pressure of the flow for $\gamma=-1.5:\left(P-P_{a t m}\right)$ vanishes on the free boundary $p=0$ and takes the maximal value on the bottom $p=p_{0}$.

\subsection{Third order}

Here, we proceed as follows: $\|\mathcal{H}\|_{2}$ and $\left\|\mathcal{B}_{0}\right\|_{2}$ for the third order asymptotic expansion are, in general, functions of $\gamma, b$ and $\tilde{B}$, see 4.1) and 4.17). So, for each value of $\gamma$, we pick the specific value of $b$ that we have obtained in the previous subsection, as illustrated in Figure 3. Then, for each pair $(\gamma, b)$, we determine the value of $\tilde{B}$ that minimizes the quantity

$$
\|\mathcal{H}\|_{2}^{2}+\left\|\mathcal{B}_{0}\right\|_{2}^{2}
$$

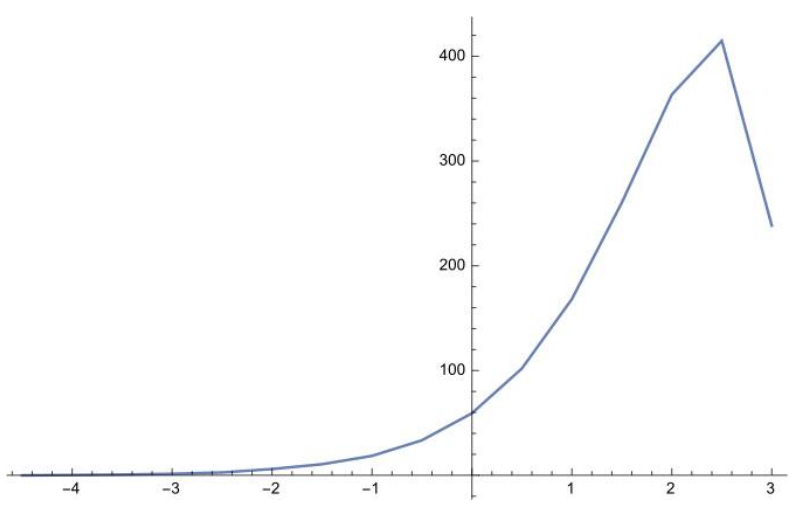

Figure 13: The value of $\tilde{B}$ for different values of vorticity, varying from $\gamma=-4.5$ to $\gamma=3$.

In Figure 13 we show how $\tilde{B}$ varies for different values of vorticity. In what follows we will display the analogue of the figures of the previous subsection, but now for the third order asymptotic expansion (4.1). 


\subsubsection{Wave profiles}

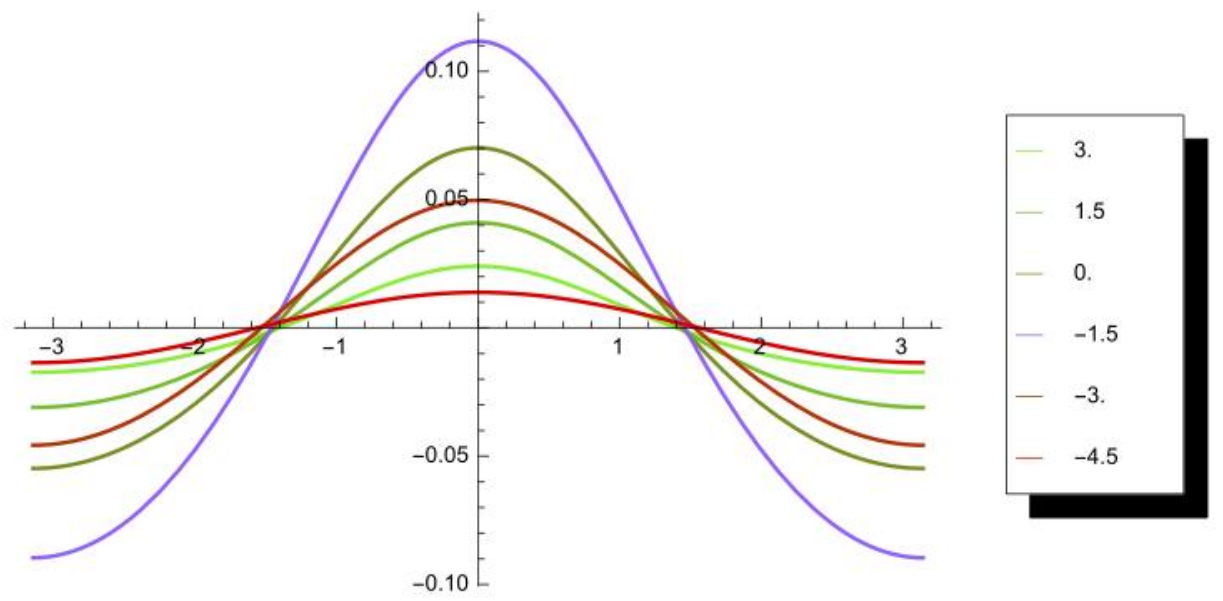

Figure 14: The free boundary for different values of vorticity, varying from $\gamma=-4.5$ to $\gamma=3$.

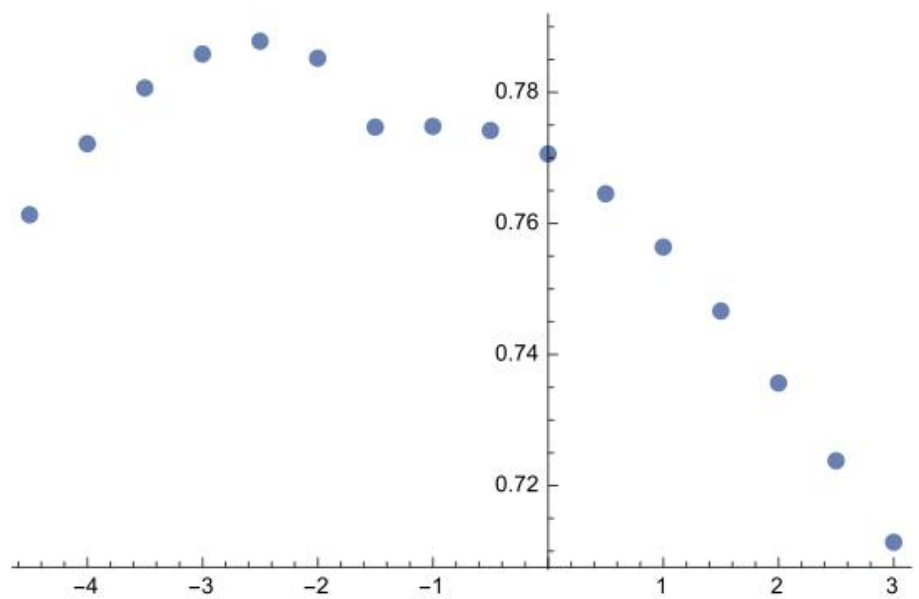

Figure 15: The mean depth for different values of vorticity, varying from $\gamma=-4.5$ to $\gamma=3$. 


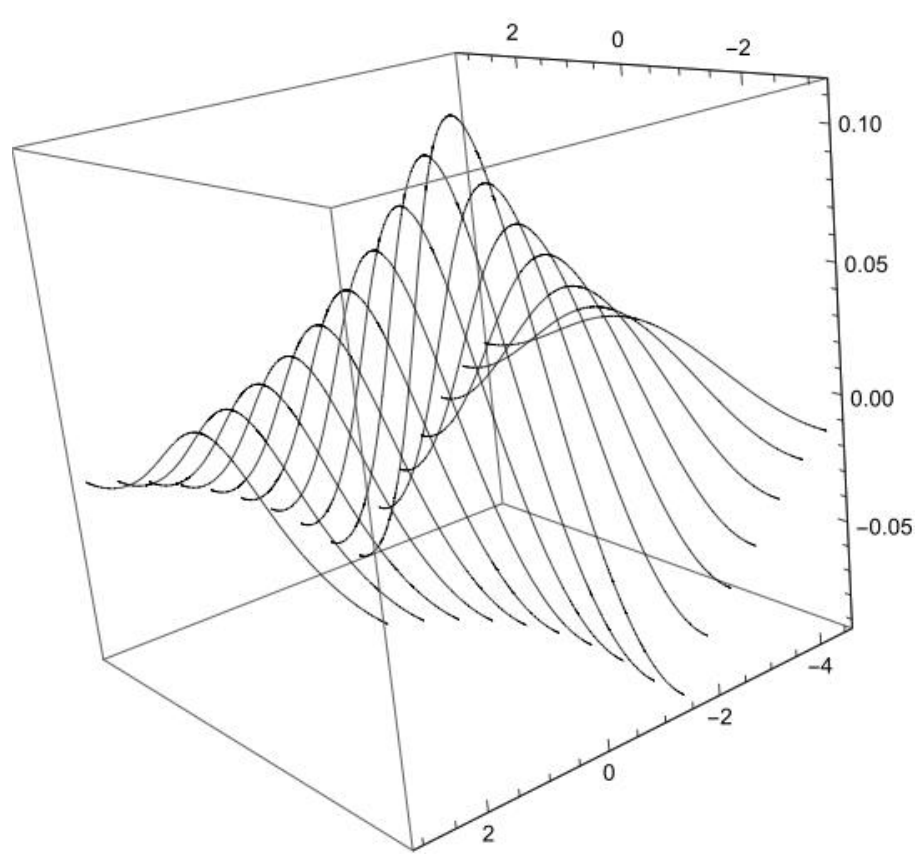

Figure 16: Each of the disjoint curves displays the wave profile $\eta(x)$, for different values of vorticity, varying from $\gamma=-4.5$ to $\gamma=3$.

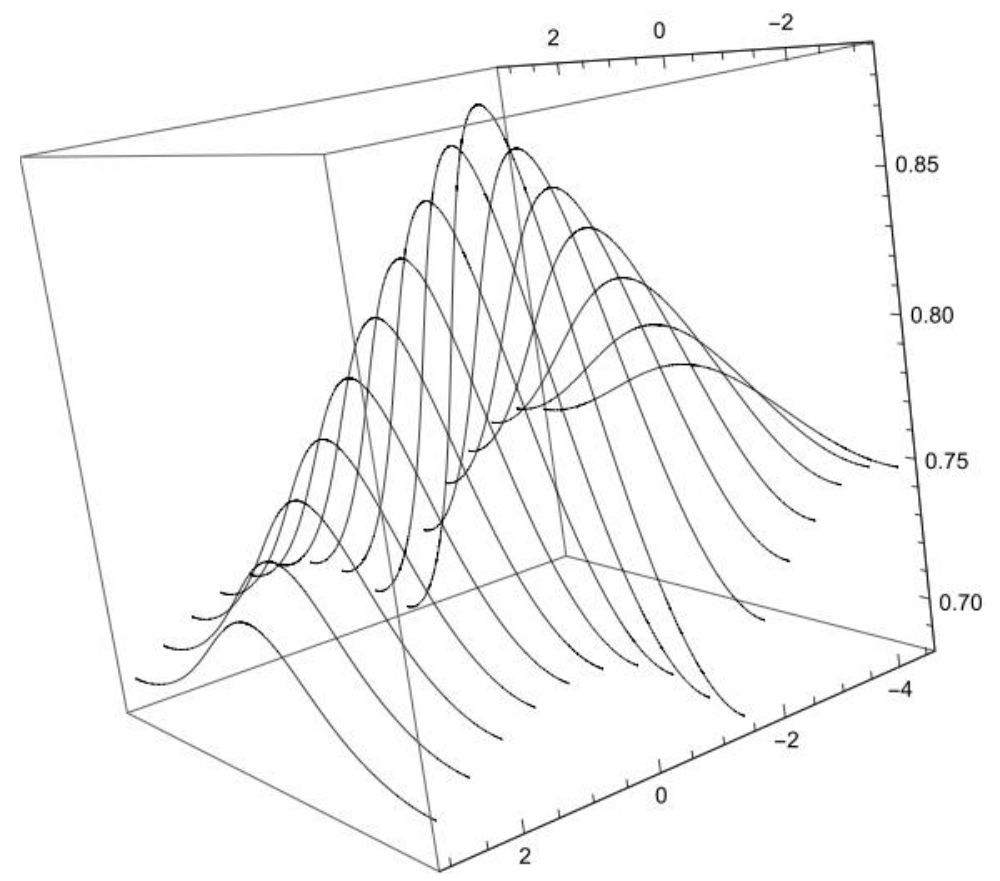

Figure 17: Each of the disjoint curves displays the free boundary $h(q, 0)=\eta(x)+d$, for different values of vorticity, varying from $\gamma=-4.5$ to $\gamma=3$. This illustration, compared to Fig. (16), takes into account the mean depth $d$, which varies with the vorticity $\gamma$, see Fig. 15. 


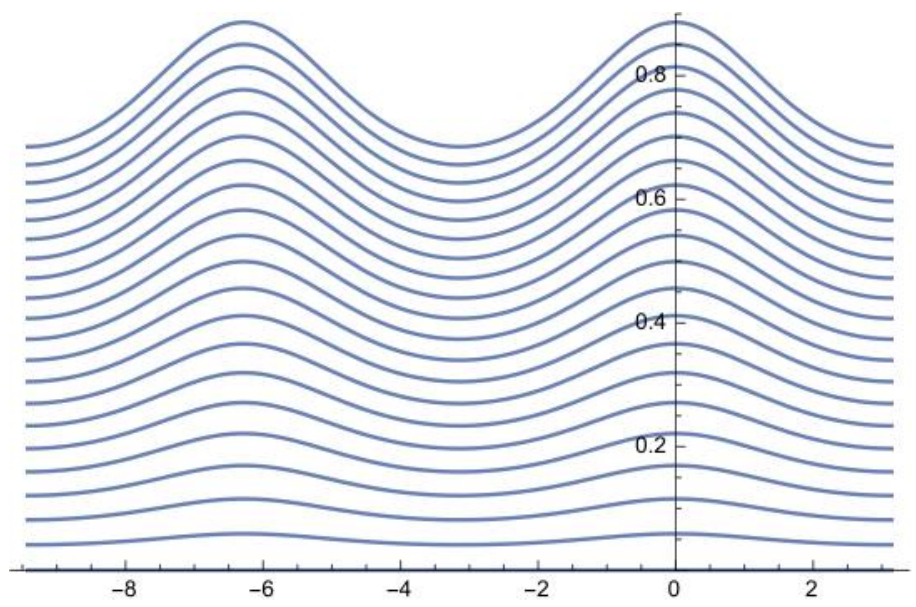

Figure 18: The height of the water along streamlines for $\gamma=-1.5$, over two wavelengths.

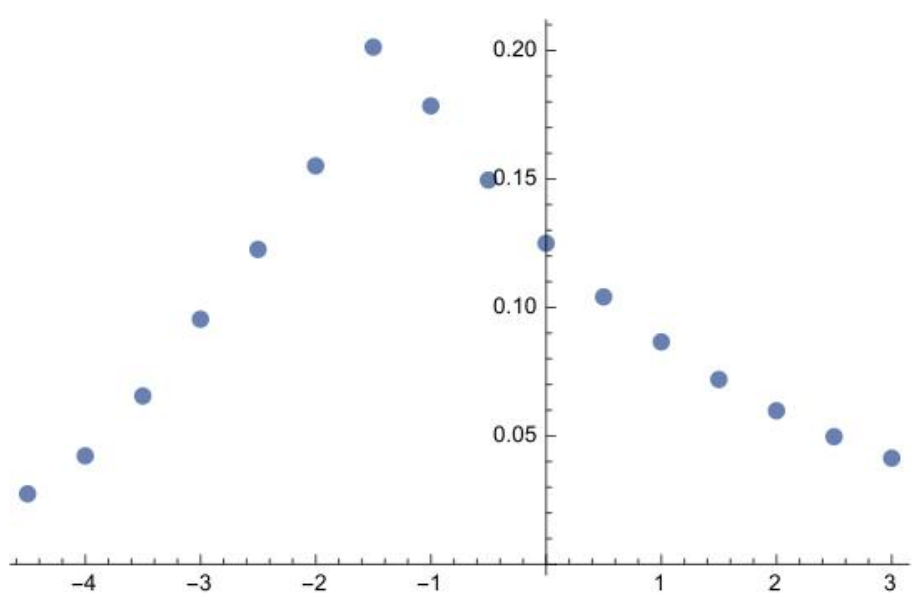

Figure 19: The wave height for different values of the vorticity, varying from $\gamma=-4.5$ to $\gamma=3$. 


\subsubsection{Velocities}

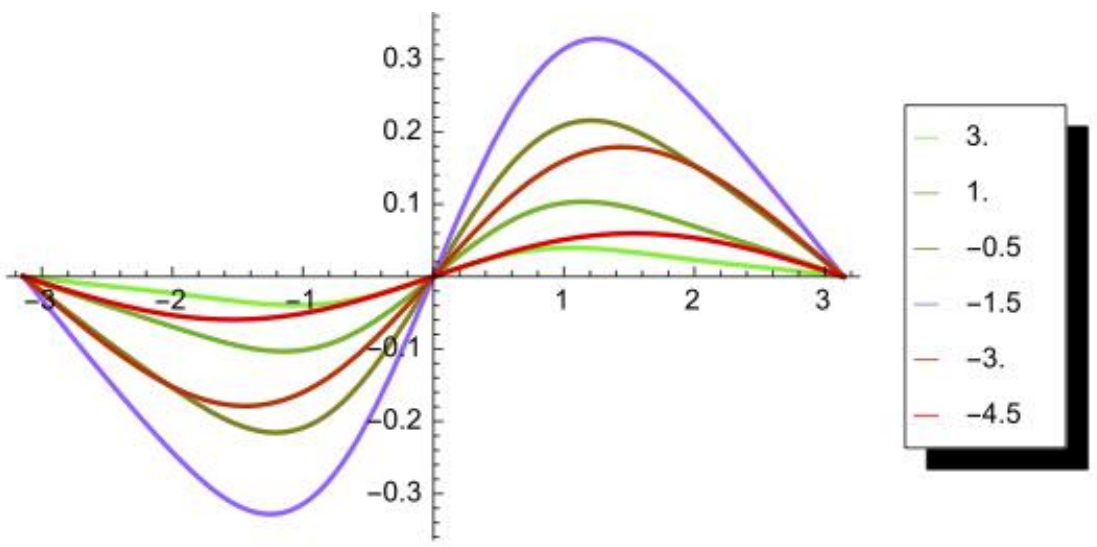

Figure 20: The vertical velocity $v$ on free surface for different values of vorticity, varying from $\gamma=-4.5$ to $\gamma=3$.

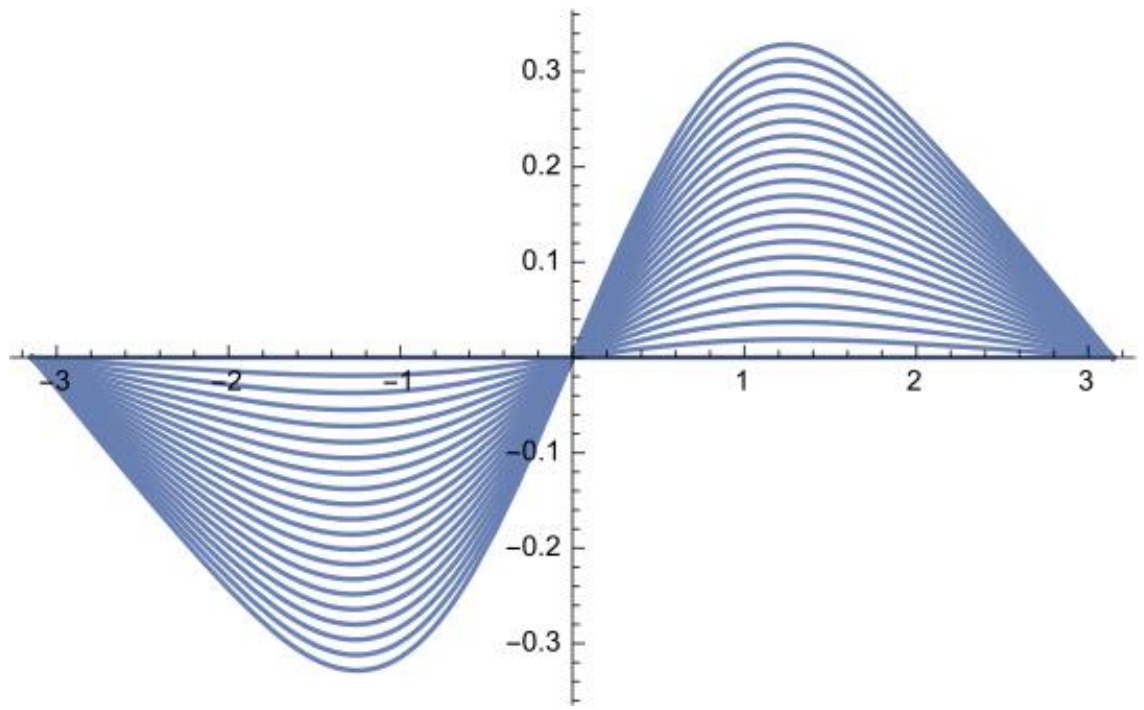

Figure 21: The vertical velocity $v$ of the flow along streamlines for $\gamma=-1.5$. 

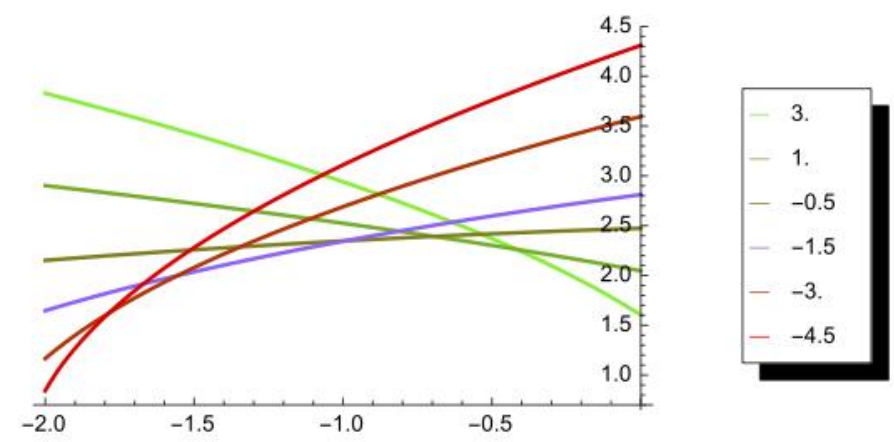

Figure 22: The horizontal velocity $c-u$ on the straight line below the crest, i.e. $q=0$ and $p \in\left[p_{0}, 0\right]$, for different values of vorticity, varying from $\gamma=-4.5$ to $\gamma=3$.

\subsubsection{Pressure}

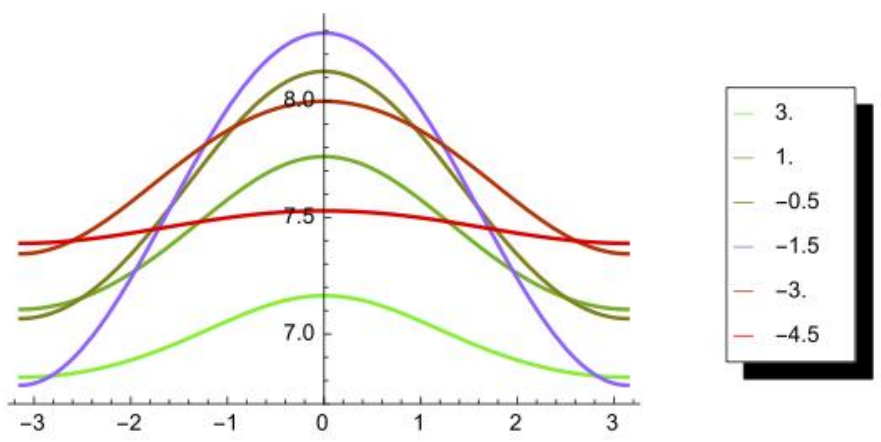

Figure 23: The water pressure on the bottom, $p=p_{0}$, for different values of vorticity, varying from $\gamma=-4.5$ to $\gamma=3$.

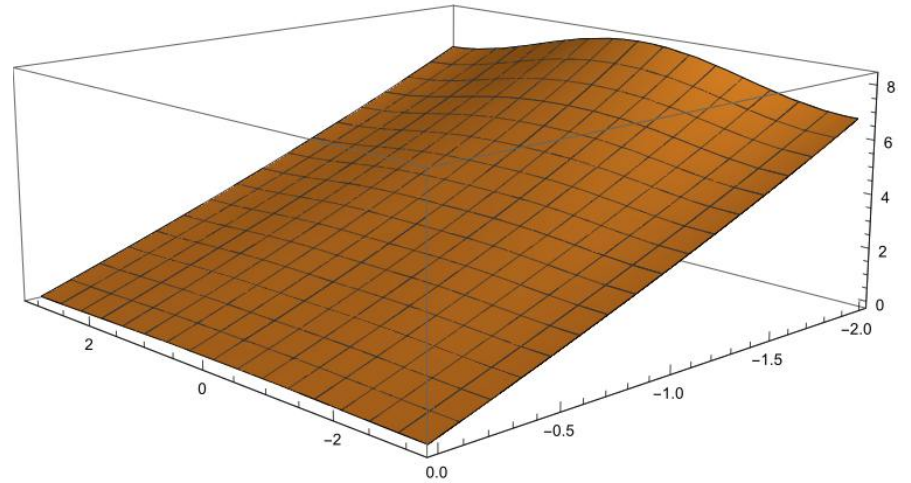

Figure 24: The water pressure of the flow for $\gamma=-1.5:\left(P-P_{a t m}\right)$ vanishes on the free boundary $p=0$ and takes the maximal value on the bottom $p=p_{0}$. 


\subsection{Comparison with existing results}

Here we compare the results that we obtained from the second order asymptotic expansion with the ones already existing in the literature, i.e. the first order asymptotic expansion. In order to choose the value of $b$ for the depiction of the first order asymptotic expansion, we proceed as in Section 5.1 and we obtain qualitative results similar to Figure 3 , but now $b$ is in general smaller, as depicted in Figure 25.

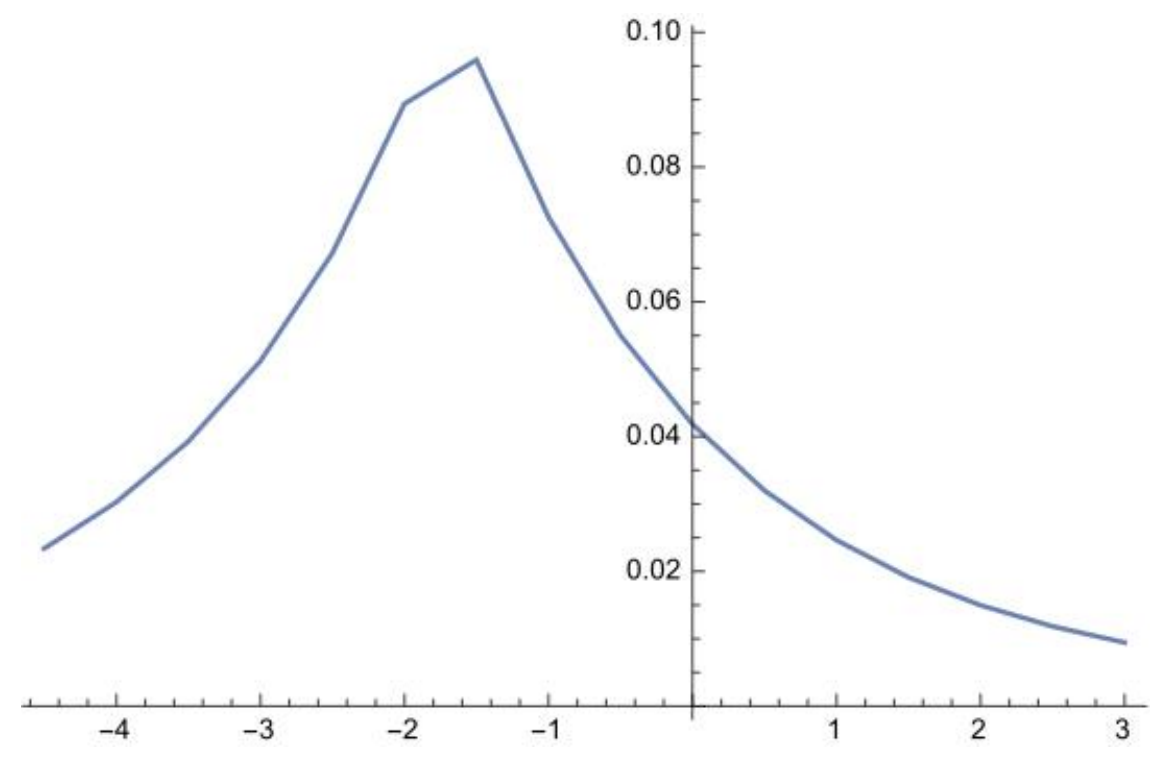

Figure 25: The value of $b$ for different values of vorticity, varying from $\gamma=-4.5$ to $\gamma=3$.

This choice of $b$ indicates the comparison on the wave height for different values of vorticity, depicted in Figure 26. There we observe that for large values of the absolute value of vorticity, $|\gamma|$, the wave height is relatively small and the first order approximation shows similar results with the second order one. However, the shape of the water profile differs significantly, see Fig. 32 and 36. Moreover, for the rest values of vorticity, we observe a discrepancy in the wave height between the two approximations. 


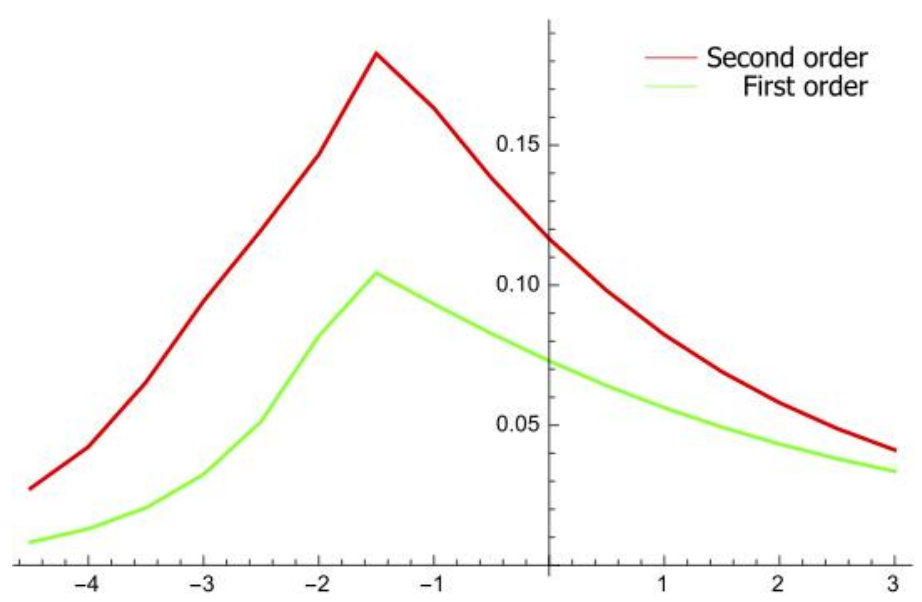

Figure 26: The height of wave for different values of vorticity, varying from $\gamma=-4.5$ to $\gamma=3$.

\subsubsection{Negative vorticity $\gamma=-1.5$}

In what follows we fix our vorticity to $\gamma=-1.5$ in order to illustrate the improvement provided by higher-order asymptotics.

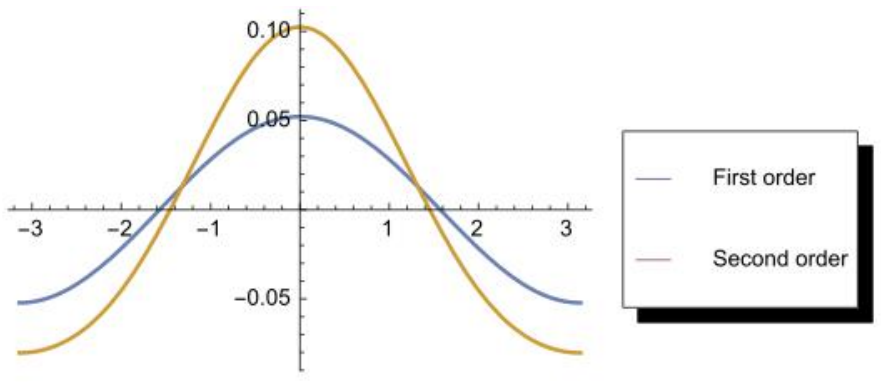

Figure 27: The free surface for vorticity $\gamma=-1.5$. The mean depth is 0.80072 and 0.79222 for the first and the second order approximation, respectively.

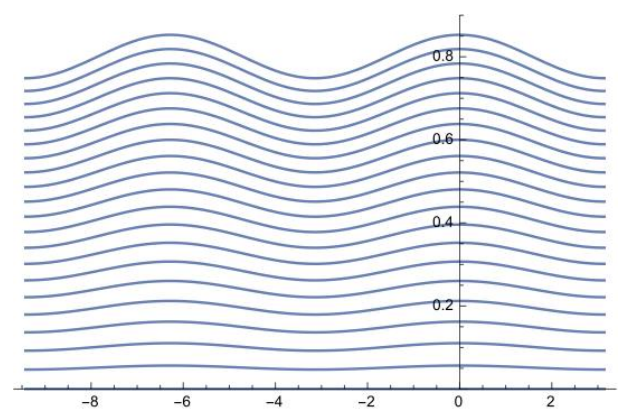

(a) First order asymptotic.

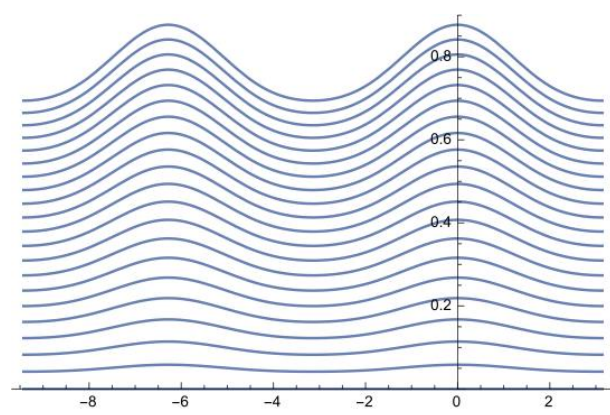

(b) Second order asymptotic.

Figure 28: The height of the water along streamlines for $\gamma=-1.5$, over two wavelengths. 


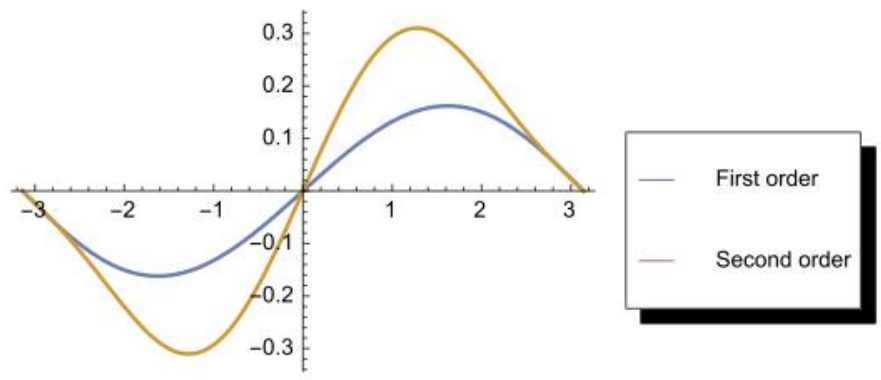

Figure 29: The vertical velocity $v$ on the free surface, for vorticity $\gamma=-1.5$.

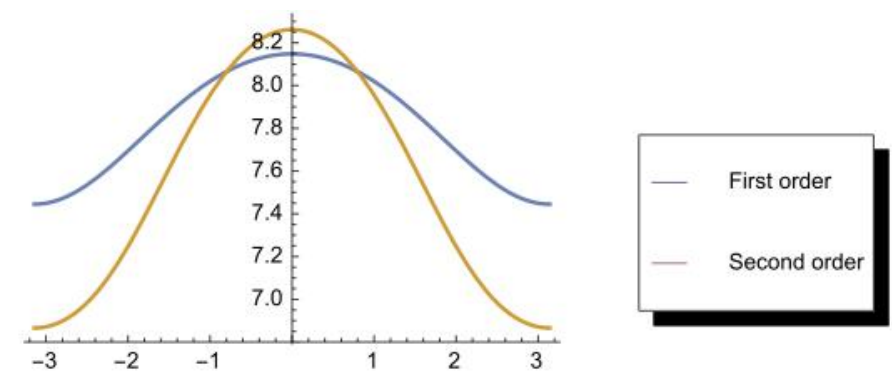

Figure 30: The water pressure: $P-P_{a t m}$ on the bottom, for the choice of vorticity $\gamma=-1.5$.

\subsubsection{Positive vorticity $\gamma=1.5$}

In what follows we fix our vorticity to $\gamma=1.5$ in order to illustrate the effect of higher-order asymptotics.

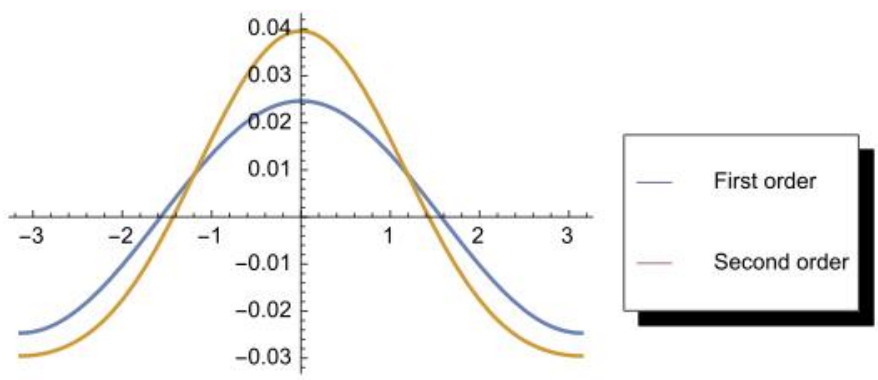

Figure 31: The free surface for vorticity $\gamma=1.5$. The mean depth is 0.758042 and 0.752228 for the first and the second order approximation, respectively. 


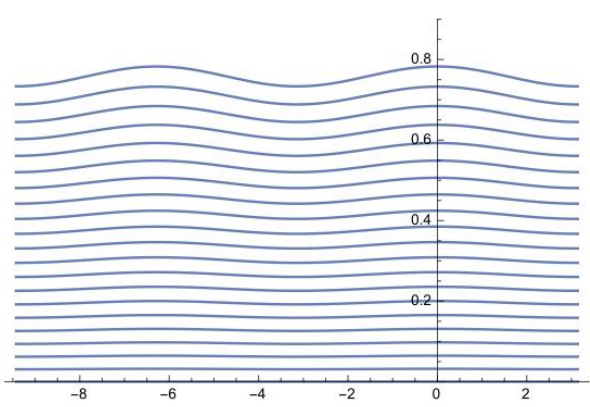

(a) First order asymptotic.

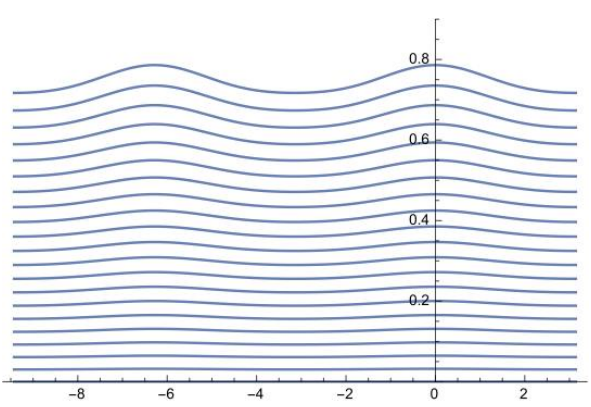

(b) Second order asymptotic.

Figure 32: The height of the water along streamlines for $\gamma=1.5$, over two wavelengths.

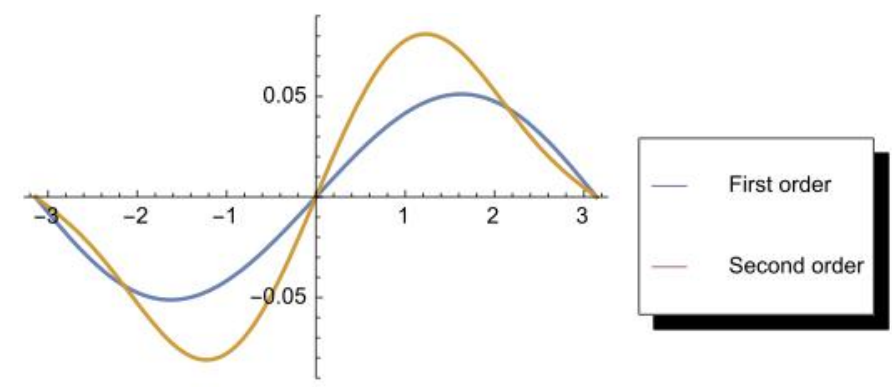

Figure 33: The vertical velocity $v$ on the free surface, for vorticity $\gamma=1.5$.

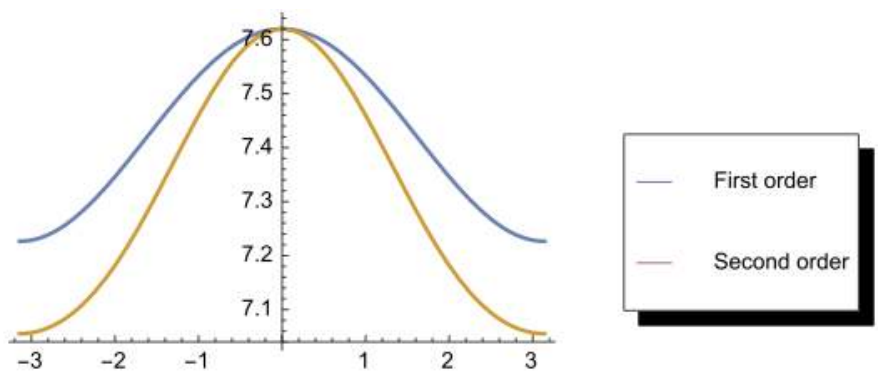

Figure 34: The water pressure: $\left(P-P_{\text {atm }}\right)$ on the bottom, for vorticity $\gamma=1.5$.

\subsection{Comparison of second and third order asymptotics}

In what follows we fix our vorticity to $\gamma=1.5$ and we compare the difference between the second and third order expansion.

In Figure 36 we depict, for different values of vorticity, the curves $h_{q q}=0$ for $p \in\left[p_{0}, 0\right]$, thus indicating where the curvature changes. Indeed, let us fix the value of vorticity $\gamma$. If for instance $p=\tilde{p}$ is fixed, then the equation

$$
\frac{\partial^{2} h(q ; \tilde{p})}{\partial q^{2}}=0
$$

has a unique solution $q=\tilde{q}$, where $\tilde{q} \in(0, \pi / 2)$. Allowing $p$ varying from $p_{0}$ to 0 , we get the 


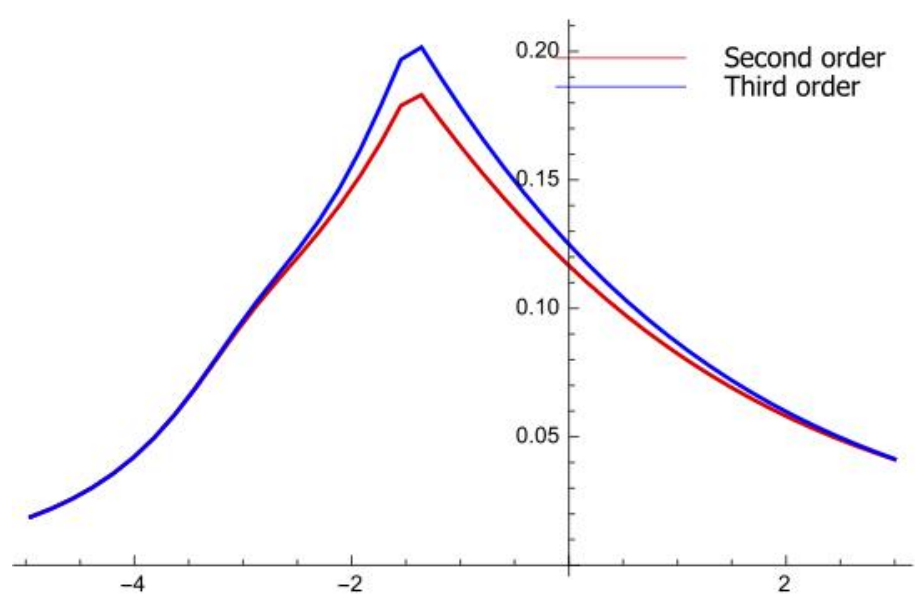

Figure 35: The wave height for different values of vorticity, varying from $\gamma=-4.5$ to $\gamma=3$.

curve $p \mapsto \tilde{q}(p)$. For different values of the vorticity we get the different curves depicted in Figure 36 , observing where the wave profile changes from convex to concave.

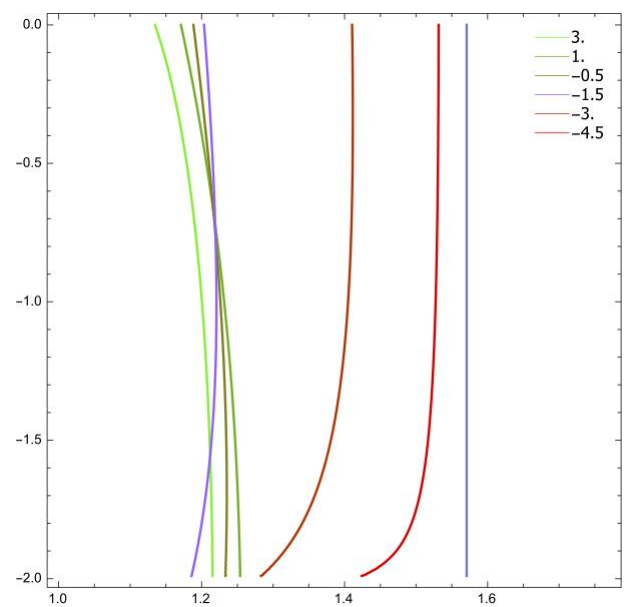

(a) Second order asymptotic.

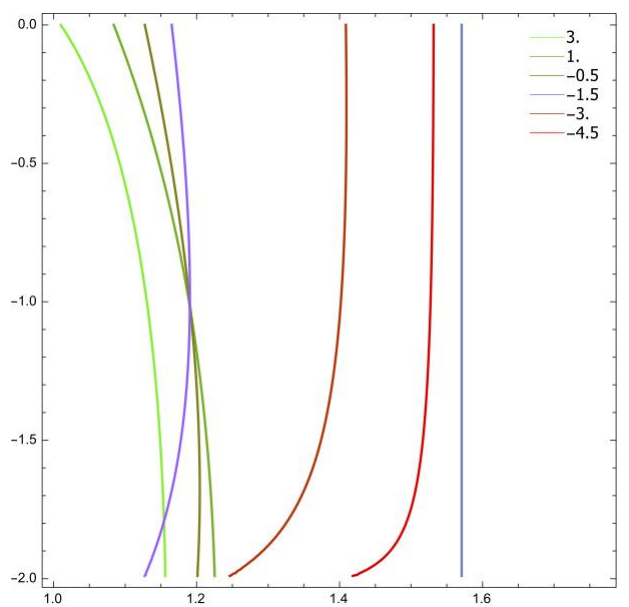

(b) Third order asymptotic.

Figure 36: The curve tracking the change of curvature, for values of the vorticity varying from $\gamma=-4.5$ to $\gamma=3$.

\section{Conclusions}

Among the conclusions that we can extract from our asymptotic expansions and the associated figures, the most important are the following:

- We observe a considerable improvement from the first to the second asymptotic expansion, noticeable in the depictions of the wave height in Fig. 26 as well as in the depictions of the profile of the wave in Fig. 28 and 32 . 
- We observe a small difference in wave height from the second to the third asymptotic expansion, cf. Fig. 35. However, there is a considerable difference in the change of the curvature, see Fig. 36 . This is due to the choice of the parameters $b$ and $\tilde{B}$. In the particular examples we did choose to keep the same values for $b$ and to minimize the errors $\|\mathcal{H}\|_{2}$ and $\left\|\mathcal{B}_{0}\right\|_{2}$, meaning that we kept approximately the same wave height and improved the accuracy of the wave profile.

- Fig. 14 shows that an opposing current has a steepening effect on the wave profile. As already pointed out in the Introduction, first-order linear theory does not capture this important feature of wave-current interactions.

Asymptotic expansions beyond those obtained here will be presented in upcoming publications.

\section{Acknowledgements}

K. Kalimeris was supported by the project Computation of large amplitude water waves $(\mathrm{P}$ 27755-N25), funded by the Austrian Science Fund (FWF). 


\section{References}

[1] Y.-Y. Chen, H.-C. Hsu and G.-Y. Chen, Lagrangian experiment and solution for irrotational finite-amplitude progressive gravity waves at uniform depth, Fluid Dyn. Res. 42 (2010), Art. 045511, 34 pp.

[2] D. Clamond, Note on the velocity and related fields of steady irrotational twodimensional surface gravity waves, Philos. Trans. Roy. Soc. London A 370 (2012), $1572-1586$.

[3] D. Clamond, New exact relations for easy recovery of steady wave profiles from bottom pressure measurements, J. Fluid Mech. 726 (2013), 547-558.

[4] D. Clamond and A. Constantin, Recovery of steady periodic wave profiles from pressure measurements at the bed, J. Fluid Mech., 714 (2013), 463-475.

[5] A. Constantin, The trajectories of particles in Stokes waves, Invent. Math. 166 (2006), $523-535$.

[6] A. Constantin, Nonlinear water waves with applications to wave-current interactions and tsunamis, CBMS-NSF Conf. Ser. Appl. Math., Vol. 81, SIAM, Philadelphia, 2011.

[7] A. Constantin, Estimating wave heights from pressure data at the bed, J. Fluid Mech. 743 (2014), R2, doi:10.1017/jfm.2014.81

[8] A. Constantin, M. Ehrnström and E. Wahlén, Symmetry of steady periodic gravity water waves with vorticity, Duke Math. J. 140 (2007), 591-603.

[9] A. Constantin and J. Escher, Symmetry of steady periodic surface water waves with vorticity, J. Fluid Mech. 498 (2004), 171-181.

[10] A. Constantin and J. Escher, Analyticity of periodic traveling free surface water waves with vorticity, Ann. of Math. 173 (2011), 559-568.

[11] A. Constantin, K. Kalimeris and O. Scherzer, A penalization method for calculating the flow beneath travelling water waves of large amplitude, submitted.

[12] A. Constantin and W. Strauss, Exact steady periodic water waves with vorticity, Comm. Pure Appl. Math. 57 (2004), 481-527.

[13] A. Constantin and W. Strauss, Pressure beneath a Stokes wave, Comm. Pure Appl. Math. 53 (2010), 533-557.

[14] A. Constantin, W. Strauss and E. Varvaruca, Global bifurcation of steady gravity water waves with critical layers, arXiv:1407.0092

[15] A. Constantin and E. Varvaruca, Steady periodic water waves with constant vorticity: regularity and local bifurcation, Arch. Ration. Mech. Anal. 199 (2011), 33-67.

[16] A. F. T. da Silva and D. H. Peregrine, Steep, steady surface waves on water of finite depth with constant vorticity, J. Fluid Mech. 195 (1988), 281-302. 
[17] M.-L. Dubreil-Jacotin, Sur la détermination rigoureuse des ondes permanentes périodiques d'ampleur finie, J. Math. Pures Appl. 13 (1934), 217-291.

[18] L. Goddijn-Murphy, D. K. Woolf and M. C. Easton, Current patterns in the Inner Sound (Pentland Firth) from underway ADCP data, J. Atmos. Ocean. Technol. 30 (2013), 96-111.

[19] D. Henry, Large amplitude steady periodic waves for fixed-depth rotational flows, Comm. Partial Diff. Eq. 38 (2013), 1015-1037.

[20] I. G. Jonsson, Wave-current interactions, In The Sea (Ed. B. Le Méhauté and D. M. Hanes), J. Wiley \& Sons, 1990, pp. 65-120.

[21] S. Kassem and H. Özkan-Haller, Forecasting the wave-current interactions at the mouth of the Columbia River, Proc. 33rd Internat. Conf. Coast. Eng., Santander (Spain), 1993, pp. 1-6.

[22] J. Ko and W. Strauss, Effect of vorticity on steady water waves, J. Fluid Mech. 608 (2008), 197-215.

[23] J. Ko and W. Strauss, Large-amplitude steady rotational water waves, Eur. J. Mech. B Fluids 27 (2008), 96-109.

[24] F. Kogelbauer, Recovery of the wave profile for irrotational periodic water waves from pressure measurements, Nonl. Anal. Real World Appl. 22 (2015), 219-224.

[25] A.-V. Matioc and B.-V. Matioc, On the symmetry of periodic gravity water waves with vorticity, Diff. Int. Eq. 26 (2013), 129-140.

[26] A. Nachbin and R. Ribeiro-Junior, A boundary integral formulation for particle trajectories in Stokes waves, Discrete Contin. Dyn. Syst. 34 (2014), 3135-3153.

[27] H. Okamoto and M. Shoji, The mathematical theory of permanent progressive water waves, World Scientific, New Jersey-London-Singapore-Hong Kong, 2001.

[28] K. L. Oliveras, V. Vasan, B. Deconinck, and D. Henderson, Recovering the water-wave profile from pressure measurements, SIAM J. Appl. Math. 72 (2012), 897-918.

[29] C. Swan, I. P. Cummins and R. L. James, An experimental study of two-dimensional surface water waves propagating on depth-varying currents, J. Fluid Mech. 428 (2001), 273-304.

[30] G. P. Thomas, Wave-current interactions: an experimental and numerical study, J. Fluid Mech. 216 (1990), 505-536.

[31] G. P. Thomas and G. Klopman, Wave-current interactions in the nearshore region, In Gravity waves in water of finite depth (Ed. J. N. Hunt), Adv. Fluid Mech., Vol. 10, 1997, pp. 215-319. 
[32] M. Umeyama, Eulerian-Lagrangian analysis for particle velocities and trajectories in a pure wave motion using particle image velocimetry, Philos. Trans. Roy. Soc. London A 370 (2012), 1687-1702.

[33] J.-M. Vanden-Broeck, Some new gravity waves in water of finite depth, Phys. Fluids 26 (1983), 2385-2387.

[34] V. Vasan and K. Oliveras, Pressure beneath a traveling wave with constant vorticity, Discrete Contin. Dyn. Syst. 34 (2014), 3219-3239.

[35] E. Wahlén, Steady water waves with a critical layer, J. Differential Equations 246 (2009), 2468-2483.

[36] J. Wolf and D. Prandle, Some observations of wave-current interaction, Coast. Eng. 37 (1999), 471-485. 